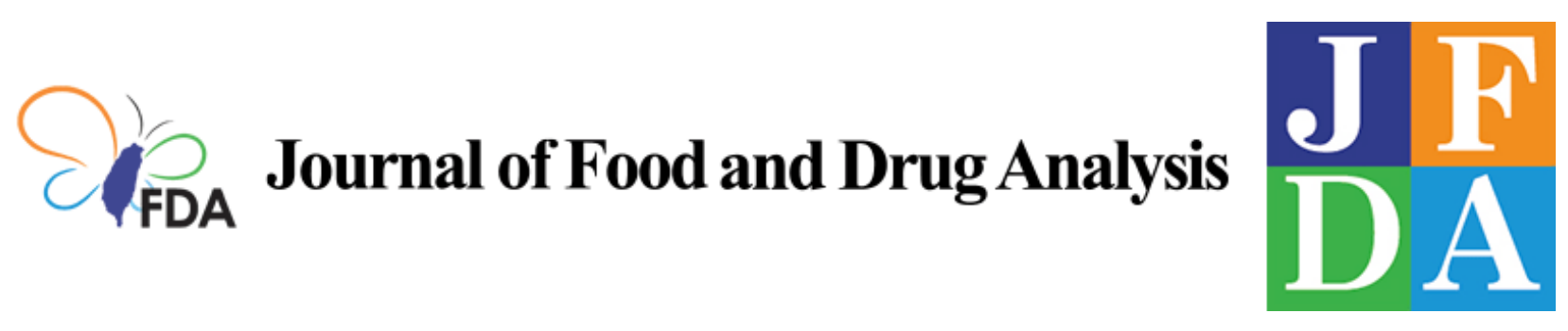

Volume 28 | Issue 4

Article 8

2020

\title{
Electrowetting-on-dielectric (EWOD): Current perspectives and applications in ensuring food safety
}

Follow this and additional works at: https://www.jfda-online.com/journal

Part of the Food Science Commons, Medicinal Chemistry and Pharmaceutics Commons, Pharmacology Commons, and the Toxicology Commons

(c) (i) (9)

This work is licensed under a Creative Commons Attribution-Noncommercial-No Derivative Works 4.0 License.

\section{Recommended Citation}

Barman, Snigdha Roy; Khan, Imran; Chatterjee, Subhodeep; Saha, Subhajit; Choi, Dukhyun; Lee, Sangmin; and Lin, Zong-Hong (2020) "Electrowetting-on-dielectric (EWOD): Current perspectives and applications in ensuring food safety," Journal of Food and Drug Analysis: Vol. 28 : Iss. 4 , Article 8.

Available at: https://doi.org/10.38212/2224-6614.1239

This Review Article is brought to you for free and open access by Journal of Food and Drug Analysis. It has been accepted for inclusion in Journal of Food and Drug Analysis by an authorized editor of Journal of Food and Drug Analysis. 


\title{
Electrowetting-on-dielectric (EWOD): Current perspectives and applications in ensuring food safety
}

\author{
Snigdha Roy Barman ${ }^{a}$, Imran Khan ${ }^{b}$, Subhodeep Chatterjee ${ }^{c}$, Subhajit Saha ${ }^{\text {, }}$ \\ Dukhyun Choi ${ }^{\mathrm{d}, * *}$, Sangmin Lee ${ }^{\mathrm{e}, * * *}$, Zong-Hong Lin ${ }^{\mathrm{a}, \mathrm{c}, \mathrm{d}, *}$
}

\footnotetext{
a Institute of Biomedical Engineering, National Tsing Hua University, Hsinchu 30013, Taiwan

${ }^{\mathrm{b}}$ Institute of NanoEngineering and Microsystems, National Tsing Hua University, Hsinchu 30013, Taiwan

${ }^{c}$ Department of Power Mechanical Engineering, National Tsing Hua University, Hsinchu 30013, Taiwan

${ }^{\mathrm{d}}$ Department of Mechanical Engineering, Kyung Hee University, Yongin, 17104, South Korea

e School of Mechanical Engineering, Chung-Ang University, Seoul 06974, South Korea
}

\begin{abstract}
Digital microfluidic (DMF) platforms have contributed immensely to the development of multifunctional lab-on-chip systems for performing complete sets of biological and analytical assays. Electrowetting-on-dielectric (EWOD) technology, due to its outstanding flexibility and integrability, has emerged as a promising candidate for such lab-on-chip applications. Triggered by an electrical stimulus, EWOD devices allow precise manipulation of single droplets along the designed electrode arrays without employing external pumps and valves, thereby enhancing the miniaturization and portability of the system towards transcending important laboratory assays in resource-limited settings. In recent years, the simple fabrication process and reprogrammable architecture of EWOD chips have led to their widespread applications in food safety analysis. Various EWOD devices have been developed for the quantitative monitoring of analytes such as food-borne pathogens, heavy metal ions, vitamins, and antioxidants, which are significant in food samples. In this paper, we reviewed the advances and developments in the design of EWOD systems for performing versatile functions starting from sample preparation to sample detection, enabling rapid and high-throughput food analysis.
\end{abstract}

Keywords: Digital microfluidics, Electrowetting-on-dielectric, Food safety, Biological assay, Lab-on-a-chip, Microelectromechanical system

\section{Introduction}

I $\mathrm{n}$ recent years, a significant number of miniaturized microfluidic devices have been developed with the ultimate goal of amalgamating entire analytical assays into a single chip-like platform to replace time-consuming conventional laboratory processes [1-5]. The primary advantages of such devices include small sample volumes, reduced reaction time, low waste generation, rapid analysis and easier sample manipulation, thereby making them an excellent choice for performing biochemical/biological studies [2,5-7]. To date, several mechanisms for driving fluid flow in microfluidic devices have been proposed such as pressure driven, capillary driven, electrokinetic flow, droplet-based and noncontact flow [8]. Irrespective of the flow mechanism, these microfluidic devices possess the common feature of integrated external components such as syringe pumps or other actuators to push the samples through the microchannels for biochemical reactions. Such external modulus-controlled continuous flow devices encounter certain challenges such as decreased mixing efficiency, difficulties with flow regulation and cross-contamination [1,4]. In addition, the fabrication of such devices is complex, and the device design is constrained to a particular application [4]. To address these limitations,

Received 26 April 2020; revised 8 June 2020; accepted 3 July 2020.

Available online 1 December 2020

* Corresponding author at: Institute of Biomedical Engineering National Tsing Hua University, Hsinchu 30013, Taiwan.

** Corresponding author at: Department of Mechanical Engineering, Kyung Hee University, Yongin, 17104, South Korea.

*** Corresponding author at: School of Mechanical Engineering, Chung-Ang University, Seoul 06974, South Korea.

E-mail addresses: dchoi@khu.ac.kr (D. Choi), slee98@cau.ac.kr (S. Lee), linzh@mx.nthu.edu.tw (Z.-H. Lin). 
various studies have recently proposed the manipulation of fluids in microchannels in the form of droplets as an alternative to continuous flow, thereby leading to the emergence of the next generation of microfluidic devices known as digital microfluidic (DMF) systems [1,4,9-11].

These digital platforms consist of a definite array of electrodes utilized for programmable fluidic manipulation at the microscale without integrating any external pumps [12-16]. Fabricated by the traditional photolithography process, DMF devices allow for multiple operations on a single platform with high precision, thereby reducing the time required for biochemical assays [8,13,17,18]. Recently, different techniques have been investigated for manipulating individual droplets in DMF devices, namely, electrowetting-on-dielectric (EWOD), dielectrophoresis (DEP), optoelectrowetting, thermocapillary forces, and acoustic wave forces $[12,15,19,20]$. EWOD provides the highest flexibility and configurability for micro- or nanolitre-sized droplet actuation controlled by an electrical stimulus, which is widely employed for labon-chip fluidic operations including creating, mixing, transporting and merging droplets.

A typical EWOD system is comprised of lithography patterned electrodes on a substrate enveloped with a thin dielectric and hydrophobic top layer $[9,21-23]$. Usually, a liquid droplet is placed in the intermediate position by the electrode embedded top plate covered with a hydrophobic layer, to which a DC or AC voltage is applied [8,24,25]. This leads to the generation of an electric field between the top electrode and the liquid droplet, changing the wettability at the dielectric/droplet interface and attracting the droplet to the surface $[24,25]$. This phenomenon is characterized by the Lippman-Young equation, where the change in contact angle is attributed to the applied voltage and hence the degree of wetting $[9,24-27]$. In recent years, the configuration of EWOD surfaces has transformed from bare to closed surfaces with the inclusion of a dielectric layer, which plays a crucial role by decreasing the leakage current between the electrodes and the droplet [28,29]. It is noteworthy that the closed configuration of the EWOD devices displayed stronger wetting performance than its bare counterpart due to the application of larger voltages [1]. Compared to other droplet actuation mechanisms, EWOD has displayed several advantages such as efficient mixing, faster reaction time, lesser risk of contamination, precise control over droplet size, enhanced sensitivity, higher selectivity, simple system design and low-cost chip fabrication, demonstrating its potential as an outstanding analytical tool for biomedical, environmental and food safety applications [1,9,30-32]. In particular, some excellent review papers have highlighted EWOD based chip fabrication protocols and sample handling methods for biomedical applications. For example, Shen et al (2014) reported the prospects of biomedical assays on portable and disposable EWOD chips based on target analytes (DNA, proteins or cells) [9]. Despite the significant advantages of EWOD systems, the integration of this technology with existing chemical and biological detection methods on smaller chip-based platforms is still the primary challenge for this technology to enter the product development phase.

Over the years, in addition to biomedical applications, EWOD-based microfluidic systems have been increasingly used for monitoring food and water contaminants for food safety applications $[9,33]$. Various reports have suggested that foodborne contaminants, especially bacterial pathogens, pose a serious threat to human health. In addition, the traditional methods for the detection of foodborne pathogens are time consuming and laborious and offer poor sensitivity that is not sufficient to detect extremely low or even single copies of bacteria present in food samples as required by regulatory standards [34-36]. Therefore, to detect a lower number of bacteria, the sample volume should be large enough to avoid any false positive results. Most of the currently available methods require pre-concentration of samples to increase the bacterial density prior to detection, hence increasing the time and cost for the analysis platform [37-40]. With the advantage of single droplet manipulation, EWOD systems stand out by further improving the limit of detection for pathogens and other food contaminant analyses. In this review paper, recent advances in the current state of the art of EWOD devices are presented for the first time for food safety applications (Fig. 1). The main focus here is to provide a comprehensive idea of the applications for chip-based EWOD devices starting from the sample preparation steps to the detection methods. In addition, this review provides readers with an imperative assessment of the potential of coupling EWOD devices with existing detection methodologies such as PCR, MALDI-TOF and MS to monitor target analytes in food and water samples with higher precision. For a clear understanding, the former sections highlight the basic concept of EWOD and the design strategies favourable for its utilization for real-time chemical/biological analyte detection, followed by applications as sample preparation modules, detection modules and other functions significant for food safety monitoring. 


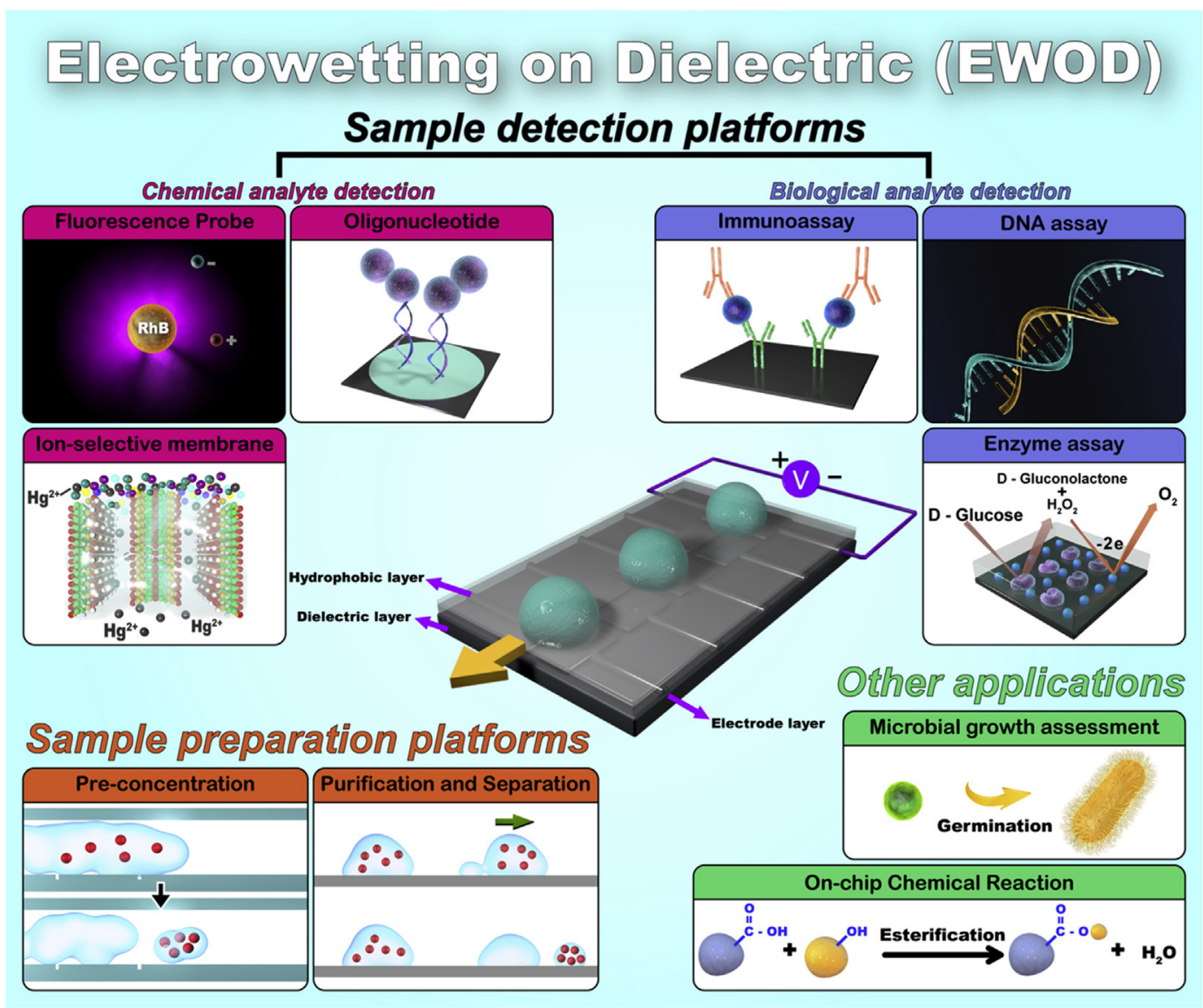

Fig. 1. Schematic representation of EWOD as a powerful food safety analysis tool. The application of the EWOD technology has been classified into three groups-sample preparation platforms, sample detection platforms and other applications.

\section{EWOD: Working principle and design parameters}

\subsection{Working principle}

The phenomenon of EWOD is based on the theory of controlling droplet movement on an electrode surface-modified with a hydrophobic dielectric layer induced by an external electrical stimulus (Fig. 2a) [9,22,41,42]. Earlier, electrowetting-based devices had problems with electrolysis even beyond few millivolts; therefore, several researchers came up with the idea of encapsulating the dielectric layer on the electrode surface, eliminating the problem and forming the principle of EWOD [43]. Utilizing a patterned electrode array, the motion of the conductive droplet can be manipulated on the solid surface without altering any chemical or material compositions. The theoretical analysis of the electrowetting phenomenon is based on the well-known Lippmann-Young's equation, stated as -

$\cos \theta=\cos \theta_{0}+\frac{1}{2} \frac{c V^{2}}{\gamma I_{V}}$ where $\theta_{0}\left({ }^{\circ}\right)$ is the initial contact angle before applying voltage, $\theta\left({ }^{\circ}\right)$ is the contact angle after applying voltage, $V$ is the applied voltage, $\gamma I v$ $\left(\mathrm{N} \cdot \mathrm{m}^{-1}\right)$ is the interfacial tension between the medium and the conductive droplet and $c$ is the difference between the capacitances of the droplet and electrode [9,42-45]. According to the equation, the application of electrical voltage leads to the accumulation of charges at the droplet-dielectric interface, changing the free energy of the dielectric, reducing the interfacial tension, and hence changing the contact angle of the surface (Fig.2b). However, the relationship between the applied voltage and change in contact angle fits well for lower voltages [46-48]. Beyond a certain higher voltage, the change in the contact angle reaches saturation $[1,47,49,50]$. This occurs because in field situations, various practical factors such as the inaccurate conduction of the droplet, ionic strength of the droplet, dielectric material, electrification of the dielectric layer, $\mathrm{pH}$ and edge effect of the capacitor contribute immensely to determining the contact angle change $[1,47]$. Studies on defining the mechanism are still 
(a)

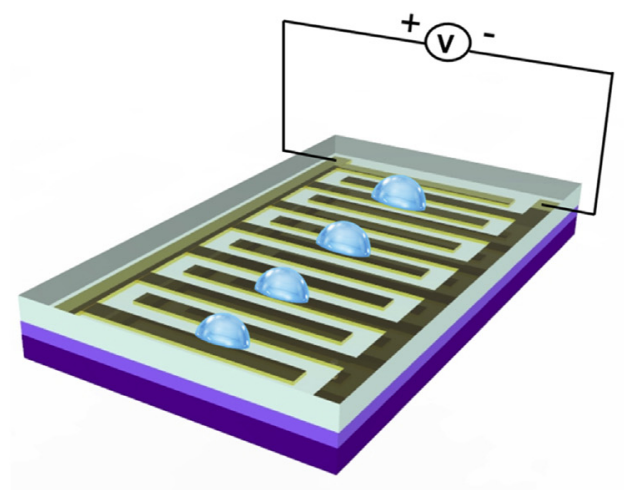

Substrate (b)
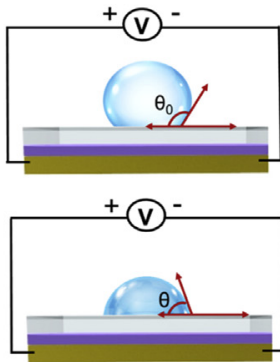

Increasing

V
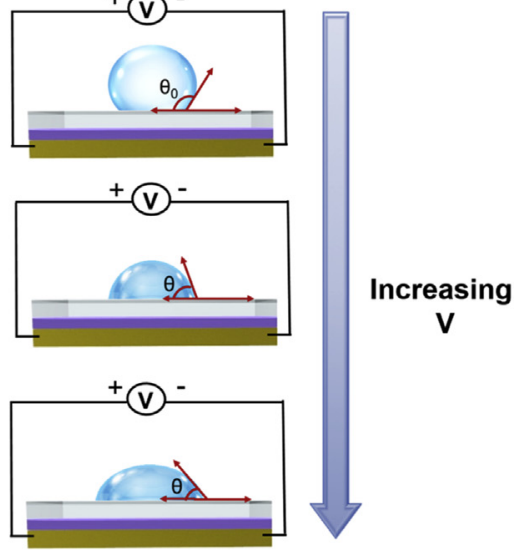

Hydrophobic $\bigcirc$ Electrode

Fig. 2. Design and performance of the EWOD chip. (a) Fabrication process of the EWOD chip. (b) Effect of applied voltage on the water contact angle.

ongoing and thus are far from reaching a conclusion.

Owing to the gap in understanding the fundamentals, since the 2000s, there has been an upsurge in the research groups around the world studying the concept of EWOD and implementing it for various applications. Since then, considerable progress has facilitated the emergence of EWOD as an important tool for droplet generation at the microscale, providing a novel perspective for digital microfluidic platforms. Various studies have reported that the type of dielectric layer, electrode configuration and applied voltage are extremely critical for designing more reliable and robust EWOD chips suitable for in-field applications [51-55]. Although recently more attention has been paid to improving the design parameters of actuators rather than solely focusing on the complexity of assays, substantial effort has also been reported on employing EWOD for applications primarily in the biomedical field $[9,56]$. The highly flexible and integrable nature of EWOD technology has been demonstrated for enzyme assays, immunoassays, DNA assays and tissue engineering $[4,57,58]$. The success of this technology in biomedical research has paved the way for applying this electrical actuation-based wetting phenomenon to other related disciplines such as food security.

\subsection{Configurations of EWOD chip}

There are two different configurations reported for the EWOD device structure-closed (parallelplane) and open (coplanar) systems. The closed configuration is a sandwich structure in which the electrode arrays are used to actuate droplets positioned on the bottom plate, whereas the reference electrodes are patterned on the bottom plate, with dielectric and hydrophobic layers deposited on the bottom plates [4,58-61]. The top plate is coated with the conductive coating and behaves as the ground electrode. Whenever an electrical voltage is applied between the top and bottom plates, the droplet moves due to the EWOD principle. On the other hand, the open configuration consists of both the actuation and reference electrodes positioned together at the bottom plate of the EWOD chip $[4,57,61,62]$. Similar to the closed configuration, dielectric and hydrophobic layers are deposited on the electrode layer, on which the droplet is placed. The closed configuration has been widely used for performing fully automated on-chip assays for food safety analysis due to its easier droplet generation and splitting ability and offers precise control over the droplet size $[58,63,64]$. Moreover, the closed design decreases the evaporation rate of the droplets from the chip surface (Min et al, 2019). Additionally, the open configuration also has their specific advantages such as the simple device design, capability to move larger sized droplets, higher mixing efficiency and compatibility with optical detection modules [65]. Although the closed system is studied by various research groups for onchip sample analysis methods, however their application is limited for off-chip sample analysis such as for MS detection and cell culture studies (Wang.J et al, 2015) [66]. In such cases, the closed EWOD chip is often integrated with its open 
counterpart, where the droplet manipulation is carried out using the closed system and the analytical detection is done using the open system [66]. Regardless of the different advantages offered by the two configurations, the basic constituents of the chip are the same. All the EWOD devices incorporate three important parts: the electrode layer, dielectric layer and hydrophobic layer. The next part of the review describes the properties of the three important layers important for fabricating electrowetting-on-dielectric chips for food safety and monitoring applications.

\subsection{Electrode layer}

The basic EWOD operations starting from the sample loading to the dispensing are usually performed with an array of electrodes. Upon application of electrical voltage to the electrodes, the surface tension of the aqueous droplet contacting the hydrophobic layer is changed, thereby driving the droplet towards the neighbouring electrode. Different materials such as gold $(\mathrm{Au})$, chromium (Cr), and indium tin oxide (ITO) are primarily explored as active electrodes fabricated by photolithography and wet etching [63,64]. However, recently, these conventional techniques have been replaced by newer methods, namely, screen printing, due to their inherent ability to reduce production time and costs [67]. Owing to their lower electrical resistance, silver $(\mathrm{Ag})$ and carbon nanotube (CNT) inks are used for printing electrode arrays for lab-on-chip applications [68,69]. Apart from the electrode material, many studies have reported that the configuration of the electrode in the EWOD chip is one of the major parameters influencing the EWOD performance [56,71,72]. For example, electrodes in an interdigitated manner resulted in higher droplet velocity leading to smoother movement owing to the enhanced driving forced originated from the improved contact length between the droplet and the electrodes [73,74]. It was observed that the droplet velocities doubled $(33.8 \mathrm{~mm} / \mathrm{s}$ to $72.7 \mathrm{~mm} / \mathrm{s})$ when the square electrodes were replaced with the interdigitated electrodes [74]. In another study, hexagon and square shaped electrode arrays were designed to achieve faster and more accurate movement of the droplet. Here, the authors suggested that the droplet manipulation is better using the square electrode pattern due to the larger contact area, which generates higher droplet velocity than the hexagon patterns [56]. Nikapitiya et al proposed two novel designs of the electrode pattern for specific droplet manipulation [75]. The L-and Y junction electrodes were designed for providing high droplet generation efficiency and rapid splitting of the droplets into uniform parts respectively. Other electrode configurations such as box-in-box patterns have also been developed for electrowetting-based sample purification platforms, which allow to-and-fro motion of the dispensing droplet from the sample site, improving the robustness of the EWOD chip [58]. In addition to the abovementioned straight edged electrodes, serpentine patterns are becoming increasingly popular for designing actuation electrodes for food and medical applications. It has been reported that the curved sides of serpentine electrodes facilitate the continuous passing of the droplet to adjacent electrodes, which is frequently blocked for straight edges [69]. Consequently, Samad et al reported that the electrode shape plays a vital role in determining the minimum actuation voltage required to move a droplet in a EWOD chip [76]. The results prove that the overlapping adjacent electrodes can displace the droplets at a lower actuation voltage compared to the square-shaped electrodes attributed to the reduced inter-electrode gap distance. Moreover, the size of the electrodes plays an important role in obtaining highly controllable droplet sizes desirable for the detection of chemical/biological analytes [77]. Despite progress, the current EWOD devices are limited by the fact that independent manipulation of droplets via individual electrodes is not possible with conventional electrode array patterns. To address this limitation, EWOD devices based on thin-film transistors (TFTs) have been proposed that consist of two dimensional arrays of electrodes that can be independently controlled for the individual manipulation of droplets in two dimensions [78].

\subsection{Dielectric layer}

One of the key elements in EWOD microfluidic chips is the dielectric layer, which separates the liquid droplet from the actuation electrodes. Choosing an accurate dielectric material is important for determining the lifetime and durability of EWOD devices [54,69,79-81]. To date, different organic materials such as Parylene $C[68,82]$ and Teflon [27] and inorganic materials such as $\mathrm{SiO}_{2}$ $[80,82,83]$ and $\mathrm{Si}_{3} \mathrm{~N}_{4}[84]$ have been widely explored as dielectrics for fabricating EWOD devices for food safety applications. However, traditional dielectric materials display certain disadvantages such as complex device fabrication, low dielectric constant and lower breakdown voltage, limiting their biological and environmental applications $[58,85]$. In recent years, novel materials such as $\mathrm{SU}-8$, which is 
a negative photoresist, have also proven to be excellent dielectric materials for EWOD devices $[55,86]$. As a photoresist, patterns can be directly made on a spin-coated SU-8 surface, which reduces the number of steps required for fabricating EWOD devices. Moreover, SU-8 possesses a relatively high breakdown voltage of $4 \mathrm{MV} / \mathrm{cm}$ and a high dielectric constant of approximately 4, suitable for operating EWOD devices [86]. Most importantly, it is reported that the difference between the actuation voltage and breakdown voltage is quite high when SU-8 is used as the dielectric layer, which improves the durability of the EWOD devices. The resilience of SU-8 to actuation allows the electrowetting platform to be reusable. Another novel dielectric material that has captured the attention of the scientific community is liquid-infused membranes (LIMs) [58]. The flexible nature of LIM dielectric materials is desirable because it can contribute immensely to making the EWOD platforms more integrable with the existing laboratory techniques, further boosting its application scope [58] LIMs, usually prepared by permeating liquids into the polymeric networks of the PTFE membranes, possess excellent dielectric properties and facilitate the reversibility of the electrowetting behaviour. The flowable liquids inside the membrane contribute to a higher breakdown voltage by allowing the movement of trapped charges to the bulk film. Furthermore, the thickness of the dielectric layer is important for optimizing the voltage required for droplet actuation using EWOD chips $[25,83]$. Ugsornrat et al found in their study that if the dielectric layer is thick, then the required voltage for actuation is high [70]. Therefore, to reduce the applied voltage, their work reduced the thickness of the dielectric layer (PDMS) by simply increasing the spin-coating speed. Typically, the thickness of the dielectric layer utilized for electrowetting-based food and biological analysis is in the range of $0.5-2 \mu \mathrm{m}$. Although a thinner dielectric layer is desirable for the majority of droplet manipulation techniques, a thicker dielectric layer is favoured for EWOD devices used to analyse liquids with higher conductivities. In a study, Kim and coworkers observed the problem of electrolysis in the EWOD device, which results in restricted droplet movement when conductive droplets are used [83]. The authors found that dielectric layers of with a thickness of $0.8 \mu \mathrm{m}$ are better than those of $0.1 \mu \mathrm{m}$ at preventing electrolysis by using a higher electric field to provide a higher actuation force.

\subsection{Hydrophobic layer}

The operation of the EWOD device relies on the application of the electric potential to change the wetting properties of the hydrophobic layer, resulting in a change in the contact angle and therefore actuating the droplet. Fluoropolymers such as Cytop are the most commonly used hydrophobic material for developing EWOD devices for both open and closed configurations [87]. In addition, other polymers, such as Teflon and Pluronics, have been explored due to their excellent hydrophobic properties by simply spin-coating a layer of a few nanometres thick [87]. Various studies have demonstrated that the surface roughness of the hydrophobic layer is crucial for determining the actuation voltage [55]. Highly hydrophobic materials with rough surface characteristics are not desirable for EWOD droplet manipulation because of the droplet-merging behaviour observed even when a higher actuation voltage is applied [69,83]. In such cases, a thin layer of lubricant is coated on the hydrophobic layer so that the droplet can move freely without sticking to the hydrophobic region. A major roadblock associated with choosing an accurate hydrophobic layer is the biofouling processes observed when biological components such as pathogenic bacteria and proteins are the target analytes $[4,88,89]$. Biofouling is a phenomenon where biological components non-specifically bind to the hydrophobic chip surface and deteriorate the performance of the electrowetting-based chip [88]. Therefore, it is important to select a hydrophobic material that can remarkably reduce the adsorption of biological samples and maintain performance for droplet manipulation [89]. For example, Kaler and co-workers designed a superhydrophobic surface with a nanostructured morphology that displayed a higher droplet angle during EWOD applications with negligible biofouling of the surface [84]. Additionally, recent progress in the field has led to the utilization of special materials such as LIMs as hydrophobic layers for droplet manipulation, where the device can operate at operating voltages as high as $350 \mathrm{~V}$ and for multiple cycles without depleting the device performance [57]. In addition to being a dielectric material, LIMs are usually filled with organic oils that are entrapped in the pores of the PTFE membrane, forming a continuous membrane that works as an excellent hydrophobic material. 


\section{Applications of EWOD}

\subsection{EWOD as sample preparation platforms}

Conventionally, the preparation of the samples for detection using microfluidic chips has been performed in separate pre-processing chambers that are not integrated with the detection systems or channels. These two-step analysis platforms are extremely complex and time consuming and contribute to higher error rates, hence decreasing the sensitivity of the detection assay. Over the years, the evolution of EWOD technology has made it possible to perform the sample processing steps prior to the detection experiments in the same chip, thereby remarkably reducing the assay time and moderating the entire process. Therefore, the following section focuses on the various salient sample preparation techniques performed on EWOD chips that have a notable influence on the food quality monitoring sector.

\subsubsection{Sample pre-concentration}

One of the problems associated with assay-based detection is that the typical concentrations of target analytes such as pathogens and metal ions present in food and water samples are quite low, making it highly challenging to detect the original concentrations without false positives [90,91]. The primary techniques available for detecting relatively small sample volumes such as the polymerase chain reaction (PCR), which relies on amplifying the sample copies, are tedious and time consuming [92-95]. In the current scenario, one approach for analysing lower concentrations of target analytes is to develop highly sensitive techniques capable of detecting considerable amounts directly from the samples. Keeping this in mind, to complement the on-chip chemical/biological assays, electrowetting-based digital microfluidic systems have also been designed to function as a sorting system for obtaining highly concentrated samples for food safety analysis [27]. For the pre-concentration process, fluorescence microparticle suspended samples were injected into a glass capillary, from which the sample entered the inlet channel of the T-shaped patterned EWOD chip positioned on the stage of a fluorescence microscope (Fig. 3a). As soon as the droplet entered the inlet channel, the EWOD electrodes were activated by the computer-controlled program to drive the droplet further to the detection channel, where the fluorescence emission signal was collected through a photomultiplier tube (PMT). Based on the fluorescence signals, the EWOD electrodes were triggered to determine the appropriate EWOD direction-either the collection zone or waste zone as shown in Fig. 3a. The photos in the upper panel displays the movement of the non-fluorescent particles towards the waste zone and the lower panel shows the advancement of the fluorescent particles towards the detection zone. In addition, the fluorescence trace signals reveal the high accuracy of sorting obtained with the asfabricated EWOD device. The authors anticipated that this device can sort and deliver approximately 10 microparticles from a sample volume of $1 \mathrm{~mL}$ in approximately 20 mins, eliminating the need for the time-consuming pre-concentrating procedures generally used for detecting low concentrations of foodborne pathogens in real samples.

The other approach relies on the two-step protocol, where the samples were first preconcentrated in a sample preparation step and then used for biological or analytical detection. Most of these systems are assimilated in a two-chamber platform; however, recently, few novel systems have been commercialized that can combine all the steps from sample collection to detection in a single tool. For instance, the Autonomous Pathogen Detection System (APDS) can couple the sample preparation stage with integrated immunoassay and PCR detection [96]. Although such systems can offer very high sensitivity, they are quite bulky and are not suitable for in-field applications. To best meet these prerequisites, EWOD has emerged as an autonomous tool to generate highly concentrated samples for further analysis in the same system, which is currently the most advanced module for on-site sampling. For instance, Foat et al reported the development of an EWOD governed air sampler to preconcentrate samples for bio-detection [97]. The key advantage of the as-developed aerosol microfluidic pre-concentrator is that the process consists of transferring the collected sample into the water droplet at the single step, thereby improving the automation and configurability of the EWOD driven sampler. In addition, the maximum concentration rate was $1.7 \times 10^{6} / \mathrm{min}$ and $6.9 \times 10^{6} / \mathrm{min}$ when operated at $5 \mathrm{~L} / \mathrm{min}$ and $20 \mathrm{~L} / \mathrm{min}$ respectively which is several orders higher than the previously developed ones. However, the charging of the collected particles by the corona needles led to the disfigurement of the hydrophobic layer of the EWOD device, leading to the decreased efficiency of the sampler. Moreover, the higher concentration of the particles led to the attachment of the particles to the hydrophobic layer of the EWOD chip, thereby changing the hydrophobicity of the device and deteriorating its functionality. Later, Coudron et al integrated a similar design for an EWOD-based 

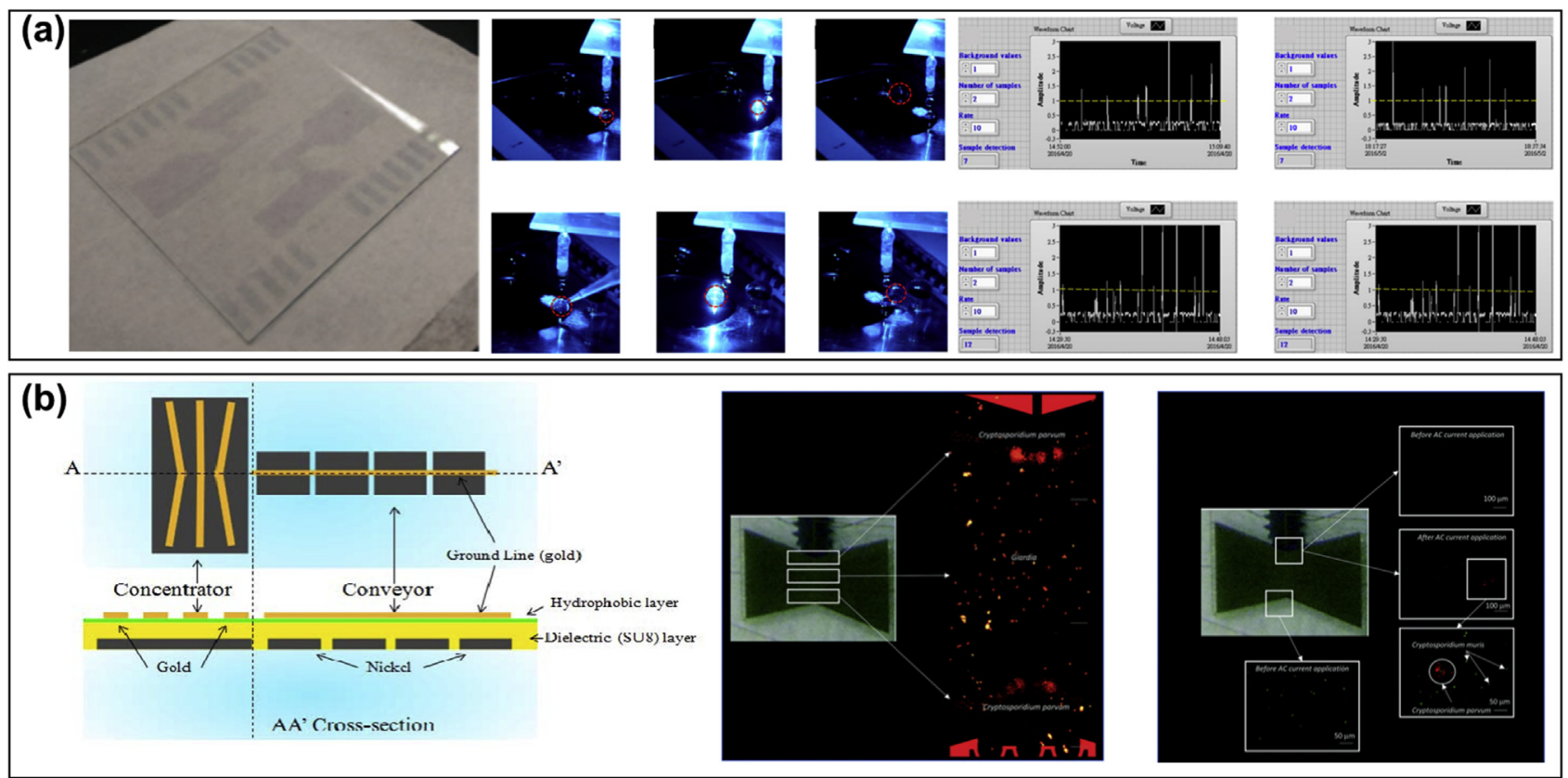

Fig. 3. Sample Pre-concentration as a sample preparation step on an EWOD platform. (a) EWOD-based microfluidic sorter for collecting fluorescent microparticles contained in the sample solution. The design layout of the EWOD chip is shown. Movement of the nonfluorescent (upper panel) and fluorescent droplet (lower panel) towards the waste and detection zone respectively and their corresponding fluorescent signals obtained from the droplets in the detection zone of the EWOD device during the sorting process. The yellow dashed lines in the upper right figure indicate the cut-off level required to actuate the EWOD chip for droplet movement. Modified and reprinted with permission [27]. Copyright 2018, MDPI. (b) Hybrid effect of EWDO-DEP for the pre-concentration of waterborne parasites prior to their analysis. Schematic of the DMF chip with EWOD and DEP electrodes and silicon cover to prevent evaporation of the samples. The selective concentration of the Cryptosporidium parvum oocysts in presence of interfering species under the applied DEP actuation of $30 \mathrm{~V}$ is also displayed. Reprinted with permission [100]. Copyright 2018, Royal Society of Chemistry.

aerosol sampler to produce highly concentrated sample droplets that were used for the detection of airborne pathogens in the same chip [82]. In addition, other methods of generating concentrated samples based on droplet sweeping mechanism in an EWOD chip have also been proposed [98]. The working of the sampler is based on the sweeping of the particles from the solid surface and their subsequent loading into the liquid medium employing the droplet driven by the EWOD actuation. For the particle concentration, the top plate of the EWOD chip was removed in order to enhance the evaporation rate, resulting in concentrated particles. The results display that the sampling was performed with high accuracy (around 90\%) even for smaller volume of samples. Moreover, the EWOD mechanism has also been combined with other DMF techniques for improving the performance of the on-chip sample preparation. Huang et al have coupled the EWOD phenomenon with DEP for preconcentrating the cell samples [99]. The cell droplet was transported to the integrated DEP electrodes with the help of EWOD actuation. The application of voltage over the DEP electrodes generated nonuniform electric fields causing the directional movement of the droplet, which was subsequently split into two highly concentrated droplets with the help of EWOD force. Although this device enabled the simultaneous manipulation of the droplets and the sample particles using the electrokinetic forces of EWOD and DEP, but their application might be limited due to the utilization of extremely high frequency for actuating the DEP electrodes. Similarly, the concentration of the droplets containing waterborne parasites for further biological detection was also done by utilizing the hybrid effect of EWOD with DEP and electroosmosis (Fig. 3b) [100]. Using the hybrid effect, the concentration efficiency was increased to $4.5 \mathrm{x}$ compared to previously reported to $1.6 \mathrm{x}$ increase when only single DMF approach was used (Fig. 3b).

\subsubsection{Sample purification and separation}

One of the most important steps prior to performing the biochemical assay or detection is the purification and separation of different kinds of biomolecules and particles which are usually carried out using external equipments such as electrophoresis, centrifugation etc, therefore limiting the practical reliability of the detection process. To 
address these constraints, various EWOD chip designs have been reported which can perform onchip sample separation of micro-particles instantaneously. Cho et al have successfully demonstrated the EWOD based binary separation of particles with the help of negatively charged carboxylate modified latex (CML) moieties and uncharged polystyrene particles. During the process, the mother droplet consisting of two different type of particles were split into two daughter droplets each containing one particle type by the EWOD actuation. Then, the asgenerated daughter droplet was driven by the EWOD voltage to the detection zone in the chip for further analysis [101]. The results indicate that the $66 \%$ and $68 \%$ of the CML droplets were separated into each daughter droplets proving successful EWOD based particle separations. Nevertheless, the formation of the vortex phenomenon was observed inside the mother droplet after sometime which made the separation process uncontrollable and decreased its effectiveness. Since EWOD displays outstanding tendency to handle liquid volumes less than $1 \mu \mathrm{L}$, it has proved to be an ideal analytical tool for the detection of analytes that exist in small quantities. These outstanding characteristic features have enabled the integration of EWOD chips with mass spectrometry (MS) for the analysis of small molecules that are limited by volume. To date, EWOD-MS coupled models have been mostly used as a sample preparation tool for the characterization of chemical and biological species present in food, water and beverage samples, rendering them important for food safety applications $[71,83,102-104]$. A novel prototype was proposed by Gorbatsova et al for MS analysis of vitamin samples in an EWOD platform that was interfaced with capillary electrophoretic (CE) separating chambers (Fig. 4a) [105]. First, the vitamin mixture solution was pipetted into the CE framework, from which small droplets were generated at the CE outlet at higher frequencies, transforming the electrophoretic chamber into an array of droplets. These droplets were then transported to the inlet of the storage tube via EWOD-based droplet actuation, where they were stored in the form of plugs for electrospray ionization-based MS (ESI-MS) analysis. Instead of the sampling tool reported in previous studies, the EWOD device played the role of collector and transporter of $\mathrm{CE}$ droplet fractions into the storage tubes. The EWOD device was fabricated in the oneplate configuration with 14 electrodes arranged in an H-shaped pattern and was activated by supplying a voltage of $100 \mathrm{~V}$ from a controllable AC source for droplet actuation. After CE separation, the EWOD platform was triggered whenever the droplet touched the electrode surface and was immediately transported to the storage inlet. Later, the storage tube was connected to the inlet of the MS detector where the mixture of vitamin solutions was investigated in the CE droplet fractions based on an ion-current strategy. The extracted ion current signals (XIC) and MS analysis proves the successful identification of the vitamins samples after droplet fractionation on the EWOD device (Fig. 4a). The primary advantage of this technique is that the droplet fraction generated was $3 \mu \mathrm{L}$ compared to the previously reported size of $12-18 \mu \mathrm{L}$, which significantly improves the MS analysis results. Even though the detection limit of this proposed technique is quite high which can be further improved by the design modifications, still this study has been a stepping stone in designing an easy and costeffective method for splitting and transporting CE output for MS analysis, eliminating the need for the conventionally used robotic controllers and expensive laboratory equipment. Similarly, EWOD based protein and peptide sample preparation for MS analysis is also reported [103,104]. For instance, Nelson et al have designed a multi-functional electrode based EWOD chip for exemplifying completely automated sample preparation for MALDI-MS analysis. Most importantly, the chip have the function of integrated heater which is used for droplet heating in order to accelerate the reaction rates. For the sample preparation, two droplets from different reservoirs were mixed and formed a combined droplet, then the combined droplet is loaded into the heating site where the sample volume is reduced due to the significant evaporation. Later, the droplet from the third reservoir was merged with the heated droplet restoring its initial volume (Fig. 4b). Insulin disulfide reduction reactions were carried out to verify the function of the coupled thermal component for sample preparation in the temperature range of $22-70{ }^{\circ} \mathrm{C}$ and the results prove that the designed EWOD chip shows high efficiency in enhancing the reaction rates (Fig. 4 b). However, in this study the effects of fluctuating temperatures during the reactions were not considered which might result in higher error rates in protein reactions where precise temperature control is desired.

In addition, other sample preparation procedures such as solid-phase extraction (SPE) commonly used in the food sector for the purification and extraction of chemical compounds suspended in liquid mixtures of foods have been combined with EWOD platforms for on-chip extraction and later analysis of the extracted chemicals using gas chromatography and mass spectrometry. Lee et al 

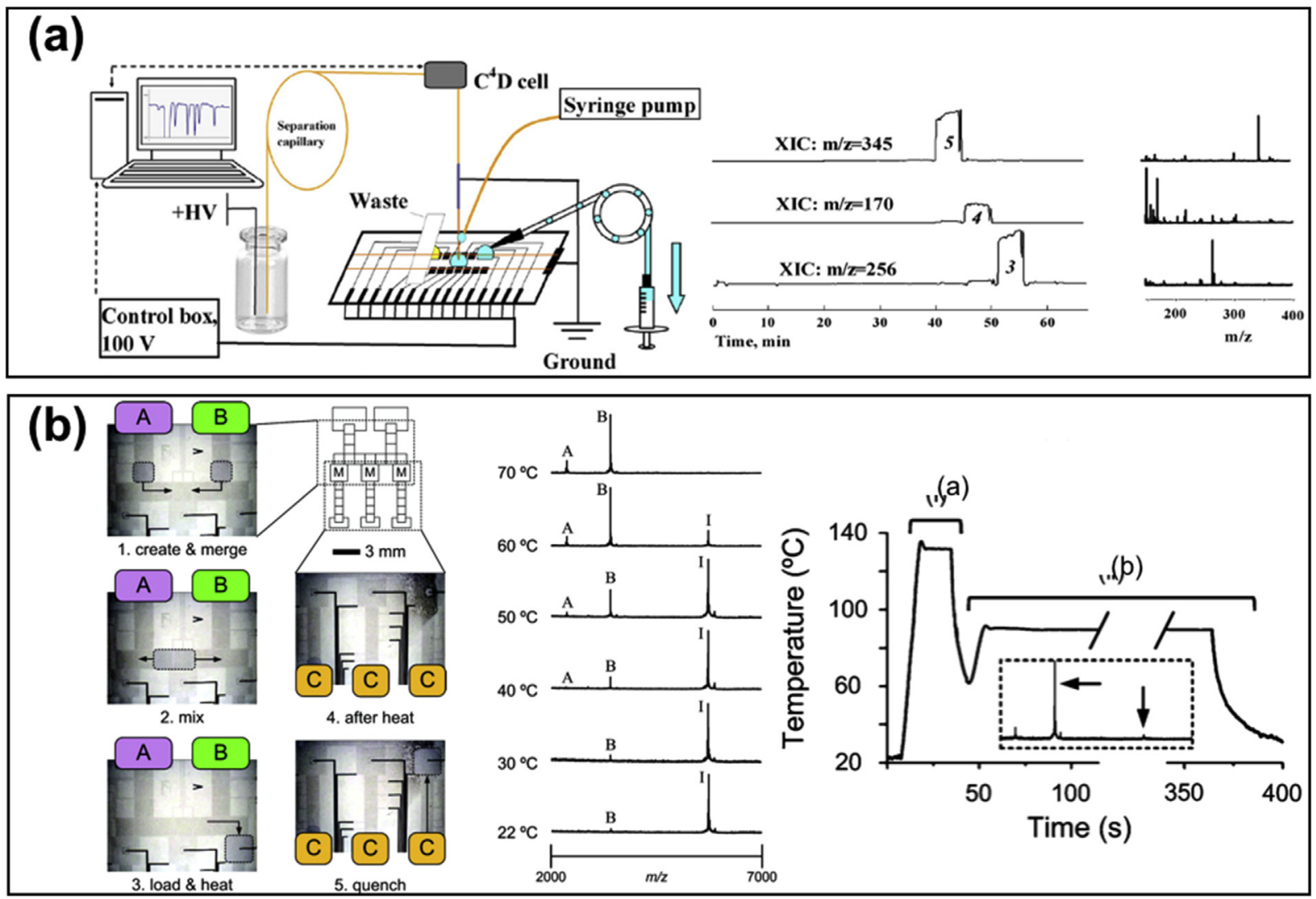
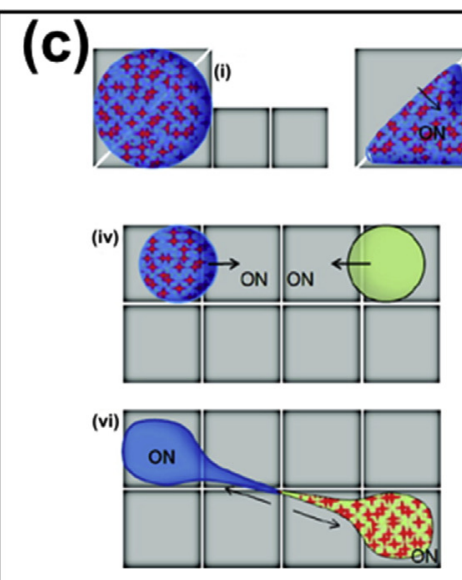

(v)
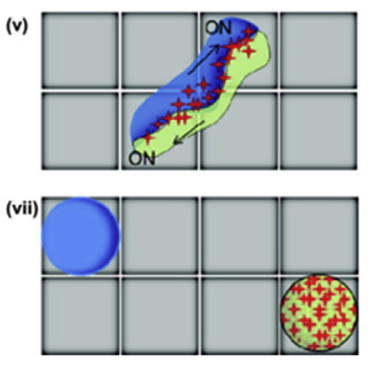

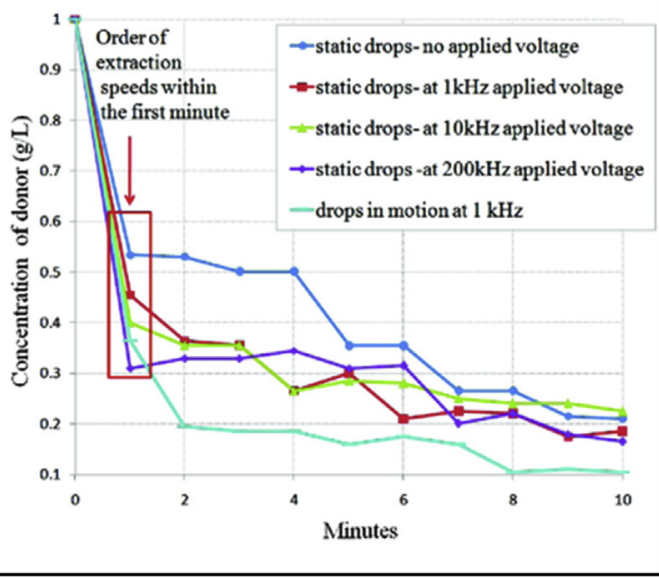

Fig. 4. EWOD as versatile sample purification platforms. (a) Integration of capillary electrophoresis-mass spectrometry (CE-MS) technology based on a highly robust EWOD chip for the off-line characterization of vitamin samples. The CE instrument was coupled with the EWOD chip for fractioning the vitamin compounds into droplets. The MS analysis of vitamin samples after the separation process using the EWOD device shows successful results. Reprinted with permission [105]. Copyright 2015, Elsevier. (b) Proteomics sample processing including protein digestion reaction on an EWOD chip for MALDI-MS analysis. The sample preparation steps displaying droplet generation, mixing, localized droplet heating and merging based on the EWOD applied voltage. In addition, the MALDI-MS spectra for insulin samples heated on chip for the insulin disulfide reactions performed in the temperature range of $22-70^{\circ} \mathrm{C}$ was obtained after the purification in the EWOD chip. Reprinted with permission [104]. Copyright 2017, Elsevier. (c) LLE of microliter samples based on EWOD actuation. The onchip LLE process is illustrated. The blue colour drop is the donor which is consists of the solute represented by red which later merges with the extractant indicated by the green colour. The relationship between the applied frequency and the extraction kinetics is also studied in which the red rectangle indicates the degree of extraction at the first minute of the extraction process. Modified and reprinted with permission [106]. Copyright 2017, Elsevier. 
successfully extracted chemical warfare agent stimulants using magnetic-bead-based SPE on a completely automated EWOD-MS device [102]. Based on the two plate configuration, the EWOD chip consisted of 14 actuation electrodes and 6 reservoir electrodes to which the sample solution was loaded. The on-chip extraction of the stimulant samples was based on the supernatant separation protocol, where the samples mixed with reaction solvents were first mixed with magnetic bead suspensions. To drive the droplet from the reservoir electrode region, a series of AC signals was applied to dispense the bead suspended sample to the collection zone for analysis using gas chromatography-mass spectrometry (GC-MS). The reservoir and the collection zone were connected using a sequence of bridge electrodes that consisted of magnets that were responsible for immobilizing the magnetic beads from the suspension; hence, only the sample in the form of supernatant was separated and passed on to the collection area. Finally, the sample obtained for GC-MS analysis was highly concentrated and enriched, unravelling a novel approach for the detection of chemical stimulants with high accuracy and importantly, minimal human intervention. Similarly, other designs of EWOD chips have also successfully demonstrated fully automated lab-on chip sample preparation for high-throughput MS analysis [71,83]. The major advantage of this integrated methodology is that onchip GC-MS detection limit significantly increased compared to the off-chip detection using the analytical instrument. However, the recovery rates for the chemical stimulants was very low which can be attributed to sticking of the analytes to the device surface and faster evaporation rate of the analytes. In addition, other studies have found that the interfacial force prevailing at the droplet meniscus in the EWOD device can increase the efficiency of the magnetic bead based collection and separation, thus making EWOD an important choice for carrying out micro-scale separation processes [105]. The results display that the collection efficiency was improved from $73 \%$ to $99 \%$ when the meniscus force was also considered along with the magnetic bead assisted separation. Not only solid-phase, but also liquid - phase extraction is demonstrated using the EWOD principle $[106,107]$. The liquid-liquid extraction (LLE) process of the acidic dye which is a solute from the aqueous droplet which behaved as the donor to the ionic liquid (extractant) was exhibited by Moon and co-workers [106]. The droplets of the immiscible liquids (donor and the extractant) were moved and driven along the electrode area using the EWOD actuation, which was finally merged and mixed for the extraction process that took place at the interface between the two droplets (Fig. 4c) Following this, the extracted phase was separated from the donor phase by fragmenting the mother droplet (single-phase) into the daughter droplets upon the completion of the extraction process. The experimental results prove that the higher frequency of the applied actuation voltage plays a vital role in generating vigorous flow patterns, thereby impacting the kinetics of the extraction process (Fig. 4c). The application of a very high frequency for improving the extraction kinetics might be a challenge for real-time sample separations procedures for food sample processing. Recently, more advanced protocol for the on-chip LLE was demonstrated by separating the specific molecules from the binary solution [107]. Moreover, for obtaining higher extraction yield, multiple cycles of the extraction process were carried out. It is clearly evident from the design and integration steps in the listed examples that the concept of sample preparation can be easily done on the EWOD platform prior to the detection of various chemical and biological analytes in the future. However, the sample preparation on EWOD chip prior to analysis is limited due to the additional steps of sample transfer which causes crosscontamination and sample loss. In addition, analysis of the separated or processed samples using the offline instruments such as MALDI-MS, GC-MS etc makes the entire process time-consuming and limits the scope for real-time applications.

\subsection{EWOD as sample detection tools}

Owing to its highly versatile and flexible nature, EWOD systems provide a promising alternative to conventional laboratory techniques for performing fully automated and controllable biochemical assays in a miniaturized and portable platform. In this section, the application of EWOD is classified based on the type of analytes to be detected, biological and chemical. In recent years, a number of EWOD systems have been developed for the detection of biological contaminants such as foodborne pathogens, proteins, vitamins, and antioxidants that are very significant for the food industry. Additionally, important chemical analytes, especially heavy metal ions and pesticides, increasingly found in food samples are determined utilizing EWOD chips. Using EWOD as the detection tool, the levels of such analytes in food were analysed with less reagent consumption, higher sensitivity, increased throughput and, importantly, without incorporating any external pumps or valves. 


\subsubsection{Detection of biological analytes}

In the last decade, EWOD-based digital microfluidics have been used in conjugation with several prevailing laboratory assays [1,3,10]. The major reason behind this is the simple and straightforward integration of EWOD systems with existing analytical procedures [108]. The type of detection assay is chosen based on the target analyte. Typically, there are three types of detection techniques: immunoassays, DNA assays and enzyme assays. The former is usually preferred for detection due to its simplicity, speed and non-complex sample preparation steps.

In 2018, Coudron et al designed a fully standalone EWOD chip for the detection of airborne pathogens such as human serum albumin (HSA), Escherichia coli (E. coli) and Bacillus atrophaeus (B. atrophaeus) that critically impacts the food sector [82]. The EWOD chip was designed based on a parallel plate configuration that consisted of 47 embedded electrodes, and the droplet was placed between the conductive cover plate located at the bottom of the EWOD chip and the hydrophobic actuation plate. The sandwiched droplet was actuated by applying a local electric field using a waveform generator, which was monitored using a microcontroller and capacitive feedback measurement system. Furthermore, an EWOD-based air sampler was coupled to produce highly concentrated sample droplets. The immunodetection was based on the binding of the target pathogen to the antibody-coated magnetic beads, which were further bound to the secondary antibody, leading to the chemiluminescent reaction between the horseradish peroxidase (HRP) conjugated on the beads and droplet luminol substrate (Fig. 5a). These droplets were used to mix the beads forming the luminescent droplets, which were actuated by EWOD, to the photodetector for detection. Therefore, rapid detection of pathogens was achieved by magnetic separation of the target pathogens with limits of detection of $30 \mathrm{ng} \mathrm{ml}^{-1}, 2 \times 10^{7} \mathrm{cfu} \mathrm{ml}^{-1}$ and $4 \times 10^{4} \mathrm{cfu} \mathrm{m}^{-1}$ for HSA, E. coli and B. atrophaeus, respectively with the average assay time of only 6 minutes for different target pathogens (Fig. 5b,c and d). Even though this work provided a good example of carrying out sample preparation and detection in the same EWOD chip, its significance is undermined by the certain challenges such as high limit of detection (LOD) and non-specific binding of the antibody-HRP complex to the magnetic beads. The authors have suggested that the replacing the current electrodes with the transparent electrode material can help to increase the sensitivity of the assay, hence the LOD by highly increasing the optical signal. In the similar manner, Liu et al have also demonstrated the ELISA process on the EWOD chip using the closed configuration of EWOD where the successful binding of the antigenantibody was detected via the electrochemical impedance spectroscopy (EIS) [109]. Besides, the mechanism of EWOD has also been combined with other detection strategies for the high-throughput analysis of important proteins and biomarkers employing the immunoassay. For example, an immunosensor based on the conventional biotinstreptavidin interaction was developed operating on the principle of DEP and EWOD. Here, the DEP was used to separate the micro particles of different sizes by application of electrical field. Further, the detection of protein functionalized on the streptavidin molecules by the biotin labelled beads were carried out by the EWOD actuation [110]. In addition, instead of traditional electrodes, specifically designed fractal capacitive electrodes were used as actuation electrodes and also as the sensing electrode for the EWOD system for the rapid immunodetection of C-reactive proteins [111].

However, if higher sensitivity is required for pathogen detection, then nucleic acid based analysis is a suitable method [112,113]. EWOD based microfluidic device consisting of 560 electrodes and 100 analysis spot patterned on the chip have been used for conducting DNA hybridization assay. The top and bottom plate of the EWOD chip consists of the negative control and the DNA complimentary to the target DNA respectively. Later, the fluorescently labelled target DNA was actuated using the EWOD principle to the detection zone for creating a concentration gradient leading to the hybridization reaction. Apart from the simple DNA hybridization assay, EWOD chips have also been used for successful demonstration of PCR reactions with enhanced sensitivity $[9,84,117-120]$. PCR is used for the detection of bacterial or viral pathogens by amplifying the target nucleic acids. Several digital microfluidic devices for PCR assays have been reported that portray certain advantages such as reduced reaction time and requirement of small sample volumes [14,114-116]. With advancements in this field, novel designs for EWOD-controlled PCR chips are proposed that ensure sample preparation and PCR detection on a single platform, which is highly desirable for in-field food sample testing. One such miniaturized design was reported by Delattre et al in 2012, where multiple pathogen samples were highly concentrated first using the magnetic bead capture method and then analysed using the PCR module [118]. In the sample preparation module, the DNA from the pathogens was 

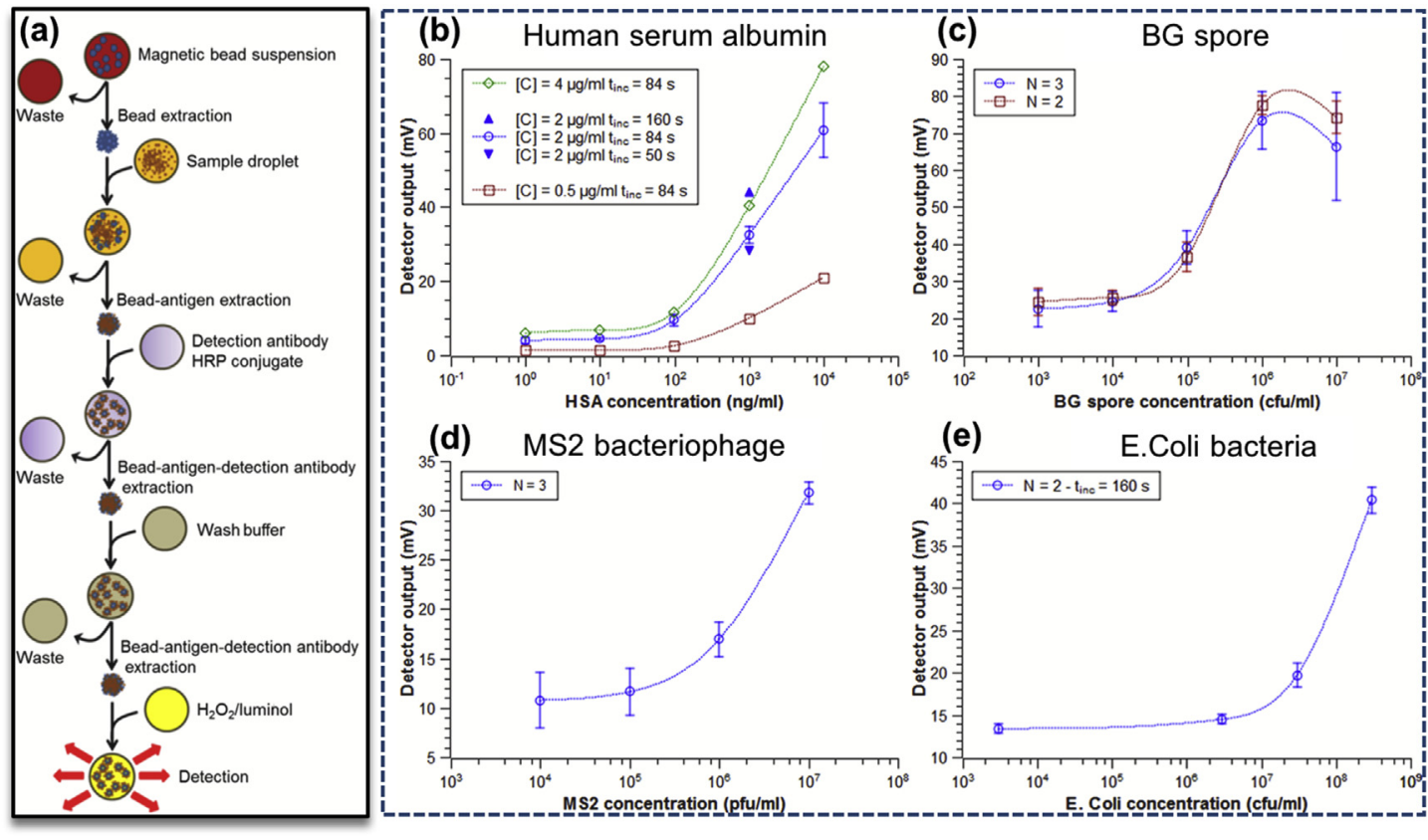

(d) MS2 bacteriophage
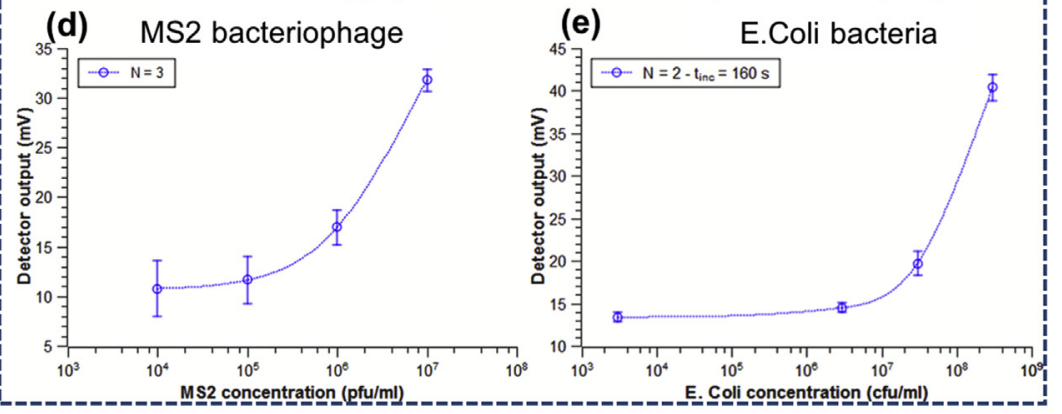

Fig. 5. Fully standalone EWOD chips are automated platforms for performing immunoassays for the rapid detection of a wide range of pathogens. (a) Process flow of the immunoassay performed on the EWOD chip using a detection antibody-HRP conjugate. Detection of different antigens - (b) human serum albumin, (c) BG spore, (d) MS2 bacteriophage and (e) E. coli bacteria using the EWOD immunoassay. Modified and reprinted with permission [82]. Copyright 2018, Elsevier.

extracted, purified and concentrated and was then mixed with the PCR reagents and pushed to the transfer channel, from which the PCR mixture was dispensed into the EWOD chip employing a pump. The PCR detection chamber consisted of an EWOD chip comprising an array of 130 electrodes and 13 reaction chambers with immobilized PCR reagents (Fig. 6a). The PCRs were performed in the form of $64 \mathrm{~nL}$ droplets that were actuated using the EWOD chip. The process of droplet PCR starting from droplet dispensing to their displacement to the mixing region comprising of embedded dry reagents is shown in Fig. 6a. The results shown in Fig. 6a clearly manifests that PCR was performed successfully on the EWOD chip for different DNA copy numbers with high accuracy. Moreover, there was no effect of increasing the cycle number on the negative control curve, proving the absence of crosscontamination between the droplets which is a key advantage. However, the sample preparation module involved the utilization of external pumps instead of any DMF based technique which made the system bulky and reduced the portability of the device. Furthermore, specific types of PCR assays known as quantitative reverse transcription polymerase chain reactions (qRT-PCRs) have been demonstrated on microchips, where PCR droplets were actuated using a combination of EWOD and electrostatic methods [84]. In this work, the sample droplet and PCR reagent droplet were mixed properly by bidirectional actuation (EWOD/DEP) on an EWOD electrode array shaped in the form of a herringbone. Specifically, the novel design of the parallelly arranged EWOD microelectrodes allowed accurate multiplexed detection of target analytes by performing multiple qRT-PCRs at the same time. Several studies have reported that PCR assay can be performed on the EWOD chip by manipulating the surface characteristics $[119,120]$. The authors have created a novel hydrophobic/hydrophilic interface to generate the surface tension gradient for actuating the droplets [119]. The key advantage of this study is that the authors have integrated the micro heater and micro temperature sensors in the channels of the EWOD chip in order to maintain the constant temperature during the PCR reaction, hence improving the efficiency of the reaction. Even so, the possibility of multiplexing for simultaneous detection of different nucleic acids have not been addressed in this work which might be a set-back. Further, valves operating on the mechanism of EWOD have also been designed and coupled with the paper-based lateral flow assays for the detection of an important foodborne pathogen Saccharomyces cerevisiae rRNA sequences (Fig. 6b) [122]. However, a major disadvantage of this study is that stronger 

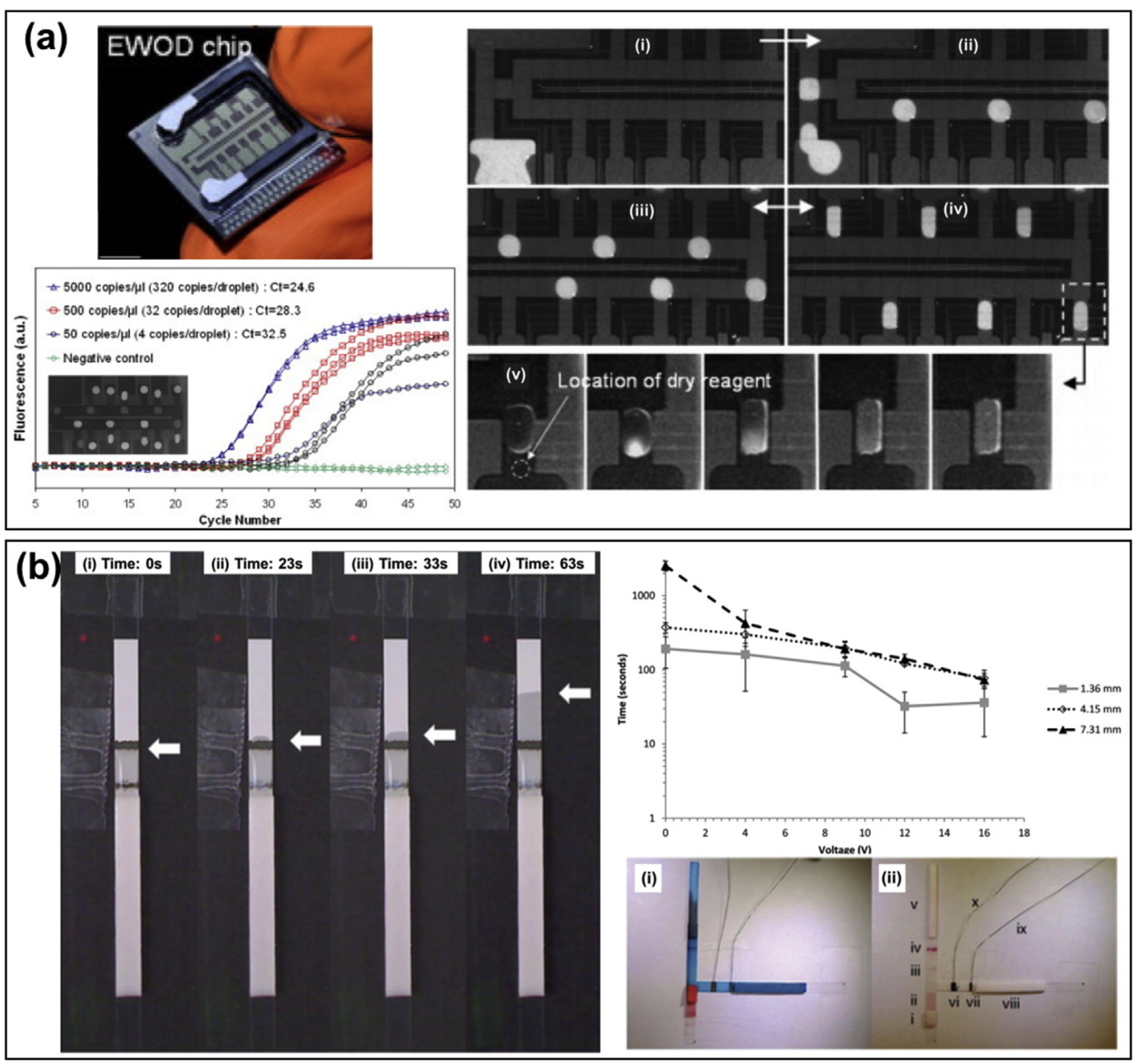

Fig. 6. Nucleic acid based detection of biological analytes driven by the EWOD actuation. (a) Polymerase chain reaction (PCR)-based detection assays were performed on EWOD chips for the identification of pathogens present in water samples. The shown design of the EWOD chip for carrying out the PCR based detection assay was operated at an actuation voltage of $60 \mathrm{~V} \mathrm{rms}$ for fluidic operations such as droplet movement and mixing. Amplification of the Baculovirus samples was demonstrated using the EWOD-PCR detection assay where the inset image indicates the fluorescent droplets when the PCR detection is completed Modified and reprinted with permission [118]. Copyright 2012, Elsevier. (b) EWOD controlled valve for the controlled fluid flow in a paper-based microfluidic device. Flow of the fluid at different time intervals on application of the potential was recorded to observed its effect on the valve opening. The results show that the time for the valve opening was decreased with the increase in the applied voltage. Finally, the as-designed electrowetting based valve was used for the detection of S. cerevisiae rRNA in lateral flow assays. Modified and reprinted with permission [122]. Copyright 2013, Royal Society of Chemistry.

fluid flow is required for washing of the samples, which might influence the lifetime of the valve. Nevertheless, a controlled fluid flow was achieved using the EWOD technique for the first time in a paper-based microfluidic device which is highly desirable for performing complex biological assays.

Apart from detecting contaminants in food, nutritional assessment of food products is of great importance for analysing the quality of samples. Identifying the sugar content (monosaccharides and disaccharides) in food products via enzymatic assays is the standard for nutritional labelling. A variety of enzymatic assays have been implemented on EWOD chips for the detection of glucose levels
[21,73,123,124]. In 2011, Vergauwe et al performed a D-glucose assay on an EWOD chip fabricated by a standard photolithography technique where the droplet was actuated by applying DC voltage [73]. The authors found that droplet size can be influenced by manipulating the electrode size, actuation time and activation time, ultimately impacting the assay performance. To avoid any discrepancies of unequal droplet size generation, a software-based controller was utilized. The quantitative detection of D-glucose was carried out by conversion of Dglucose to NADPH by using two enzymes (hexokinase and dehydrogenase) via a cascade reaction. The EWOD chip consisted of reservoirs where the 

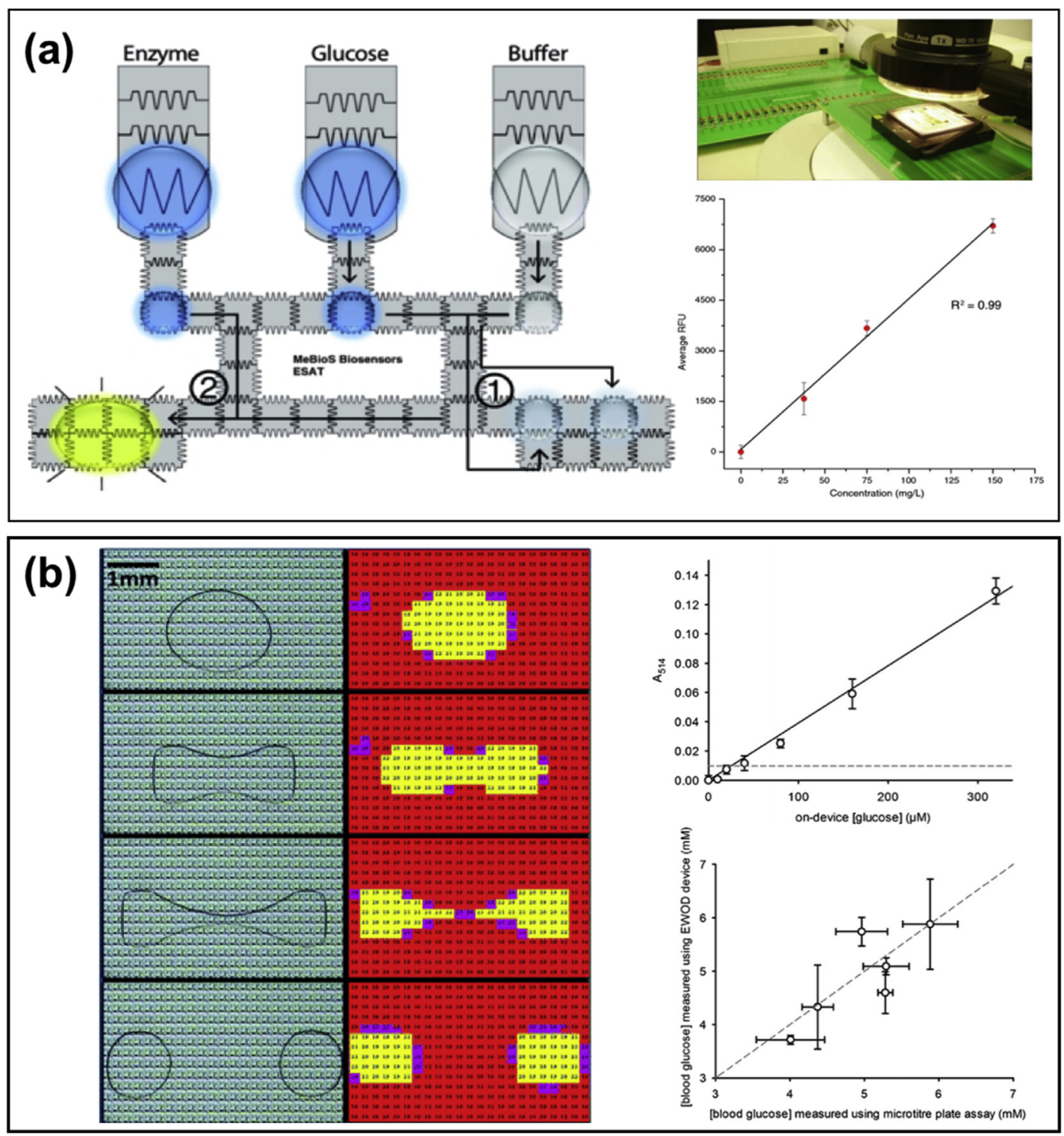

Fig. 7. Demonstration of enzymatic assays in an EWOD lab-on-a chip device. (a) Droplet based enzymatic assay for the quantitative detection of Dglucose. The chip design for performing different fluidic operations at nanoscale is shown. In the EWOD chip, dilution curves were obtained by diluting the D-glucose solution with a buffer before the enzymatic reaction. Later, the calibration curve for the diluted samples were generated for the glucose detection using the EWOD based droplet generation. Reprinted with permission [73]. Copyright 2011, IOP Publishing. (b) TFT array based EWOD device with an integrated impedance sensor for analysis of glucose samples. In the left side, the droplet based fluidic operations on the EWOD device is demonstrated where the left panel indicates the video images and the right panel represents the images from the impedance sensor. The comparison of the glucose assays performed on-chip and on the microtitre plates shows better performance of the microscale assay. Reprinted with permission [123]. Copyright 2012, Royal Society of Chemistry.

droplets of glucose and dilution solution were dispensed, merged and mixed, followed by dispensing of the enzyme droplet and then mixing with the glucose solution (Fig. 7a). The dilution curve obtained from dispensing and splitting of different sized droplets in order to generate different concentrations of glucose for the assay is shown in Fig. 7a. The limit of detection achieved was $25 \mathrm{mg} / \mathrm{L}$, and the dynamic range was $25-150 \mathrm{mg} / \mathrm{L}$, which was on par with the those of existing assays for glucose level analysis (Fig. 7a). The problem of biofouling on EWOD chips has been addressed by adding a group of triblock copolymers to the chip surface. Due to the involvement of several droplet dispensing and splitting steps, a little deviation from the required actuation leads to significant decrease in the assay performance, causing a major limitation. Further, the design of the EWOD chip was further improved for enzymatic detection by replacing the traditional electrodes with the thin 
film transistor (TFT) electrode array which allowed for independent manipulation of single electrodes (Fig. 7b) [123]. This advanced EWOD chip design contributing towards obtaining better detection limit $(5.9 \mathrm{mg} / \mathrm{L})$ which is 5 times lower than the previously reported studies (Fig. $7 \mathrm{~b}$ ). The key advantages of this work is that high flexibility of the device, inbuilt feedback control and impedance sensing functionality. In addition, during the enzymatic assay experiment, the enzymatic reagent was diluted and loaded into the chip, otherwise their performance was reduced due to the limited availability of the oxygen in the device. The aforementioned examples prove that the EWOD based sample detection platforms for biological samples have shown immense importance in the past few years in terms of decreasing the assay time and improving the overall detection performance, however the absorption of biological analytes to the EWOD device surface causing the problem of biofouling still remains a major challenge that needs to be addressed in the coming years.

\subsubsection{Detection of chemical analytes}

Chemical contaminants, namely, heavy metal ions such as mercury, cadmium, and arsenic, are highly carcinogenic and are known to have adverse effects on human health [125-129]. In particular, mercury $\left(\mathrm{Hg}^{2+}\right)$ ions bioaccumulate in the human body through the food chain and have the tendency to damage DNA and the central nervous systems, causing death [130,131]. By consuming sea foods, humans are at high risk for exposure to mercury ion contamination. Therefore, it is imperative to monitor the levels of $\mathrm{Hg}^{2+}$ ions in seafood and drinking water to prevent any chronic effects. Conventionally, the amounts of such chemical analytes are quantified using sophisticated analytical instruments such as inductively coupled plasma-mass spectrometry (ICP-MS), atomic absorption spectroscopy (AAS), and atomic fluorescence spectroscopy (AFS) [132-134]. Nevertheless, these detection methods are time-consuming, laborious, require trained professionals to operate and are not compatible with in-field detection [134]. Owing to their miniaturization, portability and high degree of flexibility, microfluidic devices are a popular alternative for evaluating the concentrations of analytes in food and water samples [135-137]. Specifically, highly controllable and fully automated EWOD-based devices that demand samples in the form of droplets to further reduce the sample volume have become efficient platforms for metal ion sensing. Most of these detection strategies are based on employing a selective probe in the form of a droplet in the EWOD chip that can specifically bind to $\mathrm{Hg}^{2+}$ ions, lowering the limit of detection. Liu et al reported an open configuration of an EWOD chip with boron-doped polysilicon as the electrode array for $\mathrm{Hg}^{2+}$ ion sensing [80]. For the selective binding of the $\mathrm{Hg}^{2+}$ ions, a complex of poly $\left(3-\left(3^{\prime}-\mathrm{N}, \mathrm{N}, \mathrm{N}-\right.\right.$ triethylamino-1'-pro-pyloxy)-4methyl-2,5-thiophene hydrochloride) (PMNT) and mercury-specific oligonucleotide (MSO) was formed by electrostatic interactions. The designed PMNTMSO probe bound specifically to $\mathrm{Hg}^{2+}$ ions through $\mathrm{T}-\mathrm{Hg}^{2+}-\mathrm{T}$ interactions, causing a colour change from red to yellow. First, a micropipette was utilized to drop the PMNT-MSO solution and $\mathrm{Hg}^{2+}$ solutions on the EWOD chip. The PMNT-MSO detector probe was fixed on the chip, and the actuation voltage was applied to generate the $\mathrm{Hg}^{2+}$ droplet and transport it to the PMNT-MSO detector probe solution, leading to the colorimetric reaction. Though this work is pioneering in demonstrating the possibility of naked eye, rapid and portable detection of $\mathrm{Hg}^{2+}$ ions in an EWOD platform, further modifications are required to lower the detection sensitivity which is currently in the micromolar.

In recent years, researchers have directed their attention towards developing EWOD chips that are more reliable for real-time detection of $\mathrm{Hg}^{2+}$ ions $[55,58]$. In the real samples, $\mathrm{Hg}^{2+}$ ions or even other metal ions differ in their chemical composition given that they are present in high saline conditions. It is noteworthy that seawater droplets have different conductivities, viscosities and permittivities, which in turn affect the EWOD actuation parameters. Therefore, amendments in the chip design were implemented by integrating a feedback loop in the EWOD chip to avoid any function failure. In 2019, Zhang et al studied the behaviour of high saline $\mathrm{Hg}^{2+}$ ion droplets in an EWOD chip for the first time [55]. Based on the traditional parallel plate configuration, the EWOD chip consisted of a patterned electrode array with 5 reservoir electrodes and 19 actuation electrodes, to which a tuneable AC voltage was applied to move the droplets (Fig. 8a). In addition, a novel feedback system based on capacitive scanning was combined with the EWOD chip to analyse the position and movement of the seawater droplet (Fig. 8b). Whenever a failure in droplet movement was observed, the capacitive charging time was increased until the droplet reached the reservoir electrodes. The detection of $\mathrm{Hg}^{2+}$ ions was carried out using a selective fluorescent probe known as 1-Rhodamine $B$ hydrazide3-phenylthiourea (RS). The sensing mechanism was based on the $\mathrm{Hg}^{2+}$ ion-induced desulfurization of 
(b)

(a)

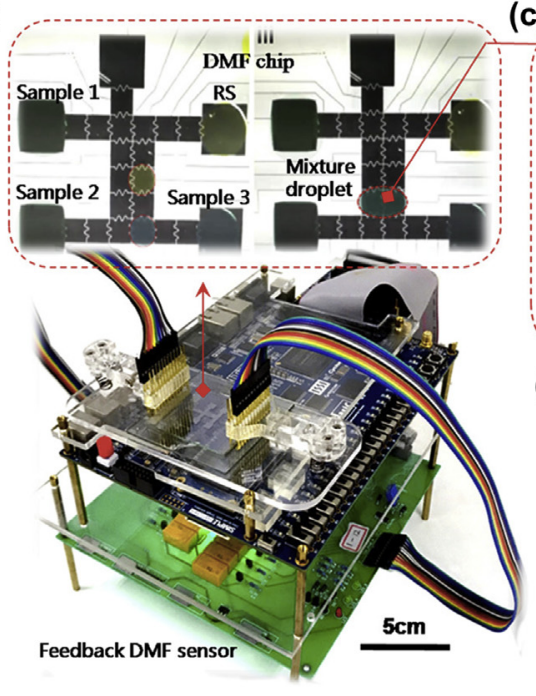

(c)

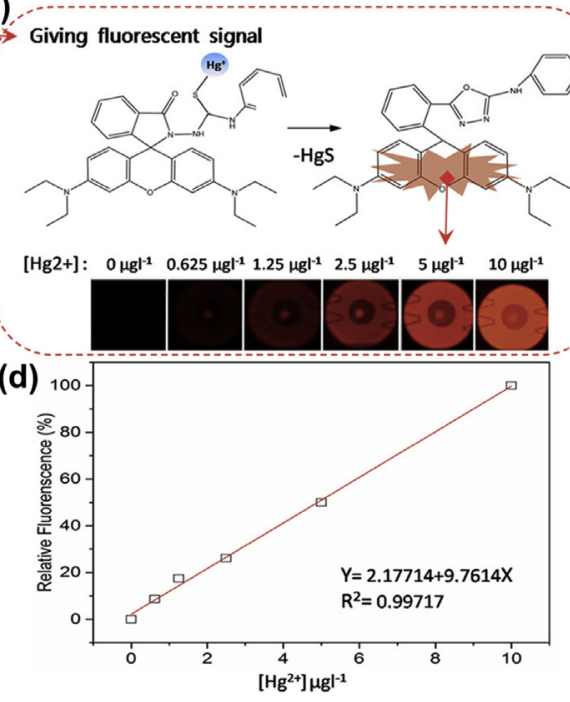

Fig. 8. Feedback-controlled EWOD technology coupled with a fluorescent sensor for the highly efficient detection of $\mathrm{Hg}^{2+}$ ions in seawater [55]. (a) Design of the EWOD sensor prototype consisting of three layers. The top layer is the chip holder, the mid layer is controller unit which can perform droplet manipulation, feedback-control and switching between the actuation mode and sensor mode. (b) Droplet operations including droplet generation, transport, mixing performed on EWOD chip for mercury detection. (c) Fluorescence probe used for the detection of $\mathrm{Hg}^{2+}$ ions in the droplets. (d) Linear range graph obtained when the RS fluorescence probe bound to different concentrations of $\mathrm{Hg}^{2+}$ ions. Reprinted with permission [55]. Copyright 2019, Elsevier.

the thiocarbonyl groups of the RS probe, causing the spirolactam ring opening transformation process. In the EWOD chip, the $\mathrm{Hg}^{2+}$ ion droplet and RS probe droplet were generated inside the reservoir electrode region, followed by merging of the $\mathrm{Hg}^{2+}$ ion droplet with the probe droplet, and finally the droplets were mixed properly to reduce the sensor response time (Fig. 8b). Later, the reaction mixture droplet was passed to the detection position, where it was detected using the fluorescence detector. As shown in Fig. $8 c$ and $d$, the fluorescence signal intensity drastically increases after reaction of $\mathrm{Hg}^{2+}$ ions with the RS probe evidencing the selective sensing of $\mathrm{Hg}^{2+}$ ions with a limit of $0.3 \mathrm{ppb}$. The major advantage of this work show that the reaction time was reduced to 3-5 $\mathrm{s}$ in the EWOD chip from the 10-15 s of the traditional fluorescence assay. In addition, the authors show that the seawater droplet displays a frequency-dependent response with a desirable range of $100 \mathrm{~Hz}$ to $1 \mathrm{kHz}$. Similar to the previous work, this study also displays a challenge in terms of the limit of detection. To detect concentrations below the limit of detection, further pretreatment of the samples is required which will in turn increase the assay time.

Although several optical sensing systems have been proposed for the highly sensitive detection of toxic metal ions, the field deployability of such prototypes still remains a major challenge. In this regard, various research groups are approaching more advanced optical designs that are easy to use and portable. One such approach is based on an optical microring resonator (OMR), operating on the principle of optical absorption measurement, which is utilized for the detection of heavy metal ions. During the sensing process, small changes in the refractive index are detected by the passing wave, which is rendered into quantifiable values such as ion concentrations. For example, Mezaine et al reported the detection of hexavalent chromium $\left(\mathrm{Cr}^{6+}\right)$ ions using the OMR technique [138]. Most importantly, the authors successfully showed the capability of combining an OMR sensing protocol with an EWOD device to further improve the portability of the sensor. The sensing principle relies on the formation of the complex between $\mathrm{Cr}^{6+}$ ions and 1,5 diphenylcarbazide (DPC), generating a colorimetric reaction that causes a change in the spectral absorption and refractive index. Cytop and SU-8 were chosen as materials for the OMR device design to ensure high compatibility for future integration with EWOD chips.

In addition to optical sensing modules, various electrochemical sensors have been added to EWOD chips for the low cost and robust detection of metal ions. Novel electrochemical sensors known as ionselective electrodes (ISEs) have emerged as efficient ion sensing tools that can be applied in different fields including the food sector. Based on the polymeric backbone, these ISEs are composed of 
ionophores that are specific molecules for sensing the target ion. Furthermore, these nanoporous membranes contain charge carriers, which make them quite simple to integrate with microfluidic systems. Min et al designed an EWOD droplet actuation-based digital microfluidic system that employed ISE sensors for the detection of ammonium $\left(\mathrm{NH}_{4}^{+}\right)$ions from a mixture of interference ion solutions [69]. The use of fertilizers and pesticides raises the amount of $\mathrm{NH}_{4}^{+}$in the soil, inducing toxicity in grain crops and resulting in environmental pollution. In this work, the microfluidic chip for driving the droplet consisted of an ISE sensor, microcontroller, voltage supplying cell and capacitanceto-digital convertor (CDC). An array of eight electrodes was designed in a serpentine pattern on the EWOD chip, which included a region for droplet initiation and the ISE sensing region (Fig. 9a). First, droplets were placed at two different points inside the droplet initiation region and moved towards each other; the droplets then mixed and proceeded to the sensing area. In order to facilitate the droplet movement without the droplet merging characteristics, silicone oil was used as a lubricant for the hydrophobic layer. After applying the silicon oil, the smooth movement of the droplet towards the sensing area was observed as shown in Fig. 9b. The droplets were driven using a voltage supplying cell that could amplify the lower voltage to the higher values required for droplet manipulation. The system also integrated a capacitance sensing-based feedback loop to monitor the movement of the droplet employing the CDC system. The results obtained showed highly controlled droplet generation capability along with precise speed control. This advanced design demonstrated high sensitivity towards the target ion, i.e., $\mathrm{NH}_{4}^{+}$ions in the dynamic range from $10^{-6}-1 \mathrm{M}$ (Fig. 9c). In addition, the membrane shows excellent selectivity towards detection of $\mathrm{NH}_{4}^{+}$ions even in presence of interference ions, $\mathrm{K}^{+}$and $\mathrm{Ca}^{2+}$ (Fig. 9d). However, in presence of the interference ions $\mathrm{K}^{+}$, the linear response of the as-developed sensor decreases due to the dominance of the interference ion which forms a major disadvantage. Furthermore, analytes such as L-ascorbic acid and nicotinamide adenine dinucleotide, significant for food technology, were detected electrochemically by employing a disposable EWOD chip. The EWOD chip contained screen printed electrodes arranged in the $\mathrm{T}$-junction, integrated with the working, counter and reference electrodes for electrochemical detection located at the end of the T-junction. Due to the EWOD actuation, the analyte and buffer droplet were mixed rapidly at different concentrations for immediate detection using the electrochemical detector. The results obtained display comparable sensing performance with a total analysis time less than $14 \mathrm{~s}$,
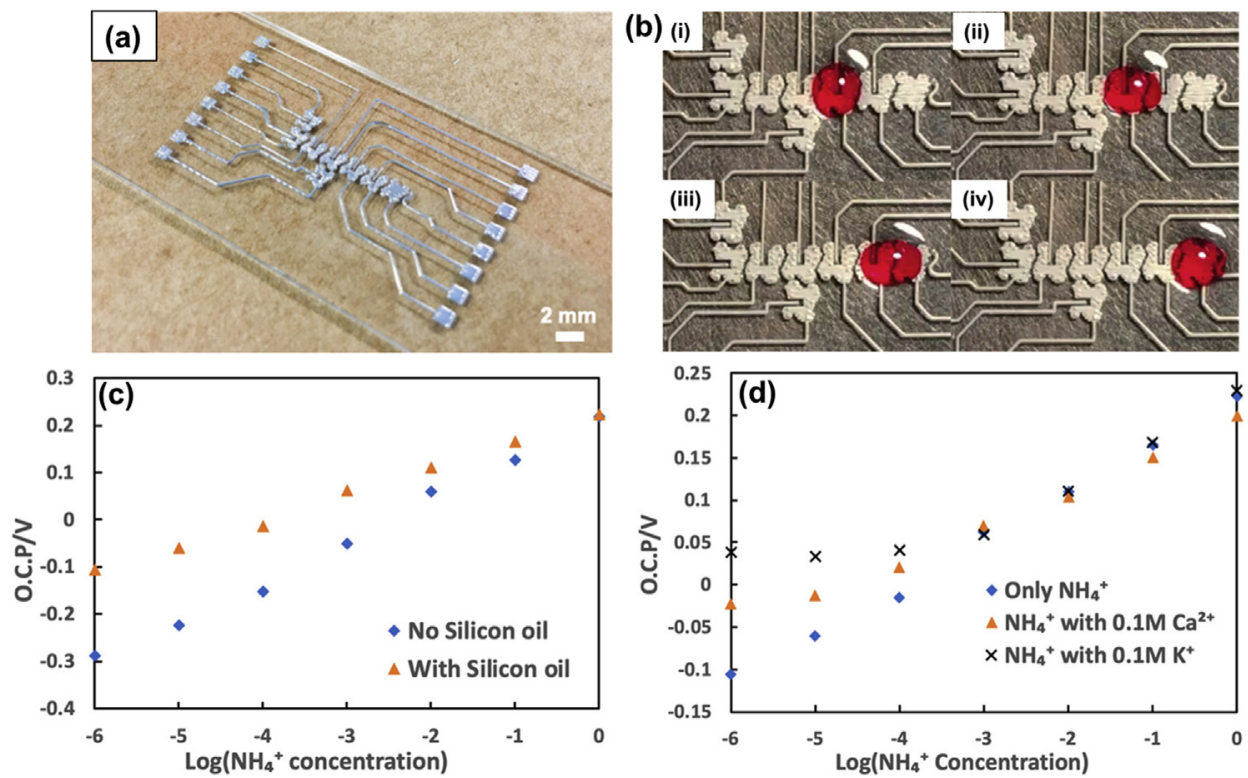

Fig. 9. EWOD-governed ion-selective sensing systems for the selective detection of $\mathrm{NH}^{4+}$ ions from a mixture with interference ions. (a) Design of the printed EWOD chip. (b) Movement of the droplet towards the sensing area. (c) Open circuit voltage generated during the detection of different concentrations of $\mathrm{NH}^{4+}$ ions at using the ISM coated on the EWOD chip with and without the silicon oil. (d) Selectivity study of ISM towards $\mathrm{NH}^{4+}$ ions in the presence of interference ions $\mathrm{Ca}^{2+}$ and $\mathrm{K}^{+}$. Modified and reprinted with permission [69]. Copyright 2019, American Chemical Society. 
suggesting an attractive alternative for low-cost chemical analysis. The detection of chemical analytes on EWOD chip have displayed rapid response and high configurability for real-time detection, however before commercialization of such devices, the limitation of the detection sensitivity needs to be resolved so that the sensor is at par with other optical based chemical sensors.

\subsection{Other applications}

Over the years, researchers have started to explore EWOD platforms to conduct on-chip chemical reactions because such platforms allow for precise control of droplet generation and mixing and prevent any cross-contamination due to undesired diffusion. In addition, EWOD systems abolish the necessity of using mechanical pumps and valves, thereby making them cost-effective compared to other microfluidic reactors [64,139-142]. Each droplet generated acts as a batch reactor, which allows for simultaneous multiple reaction steps suitable for combinatorial chemistry
$[53,140]$. Vaultier and co-workers have demonstrated the on-chip chemical reaction utilizing ionic liquids displaced by the EWOD actuation (Fig. 10a) [139]. Room-temperature ionic liquids (RSILs) were moved and mixed with other task-specific ionic liquids (TSILs) droplets, each containing different reagents for carrying out the organic synthesis of tetrahydroquinolines. This work displayed for the first time that not only aqueous liquids but also ionic liquids can be subjected to EWOD actuation. Moreover, the negligible volatile nature of ionic liquids allowed movement of the low-volume droplet in an open-type EWOD chip without using any top cover or oil. The comparison of the chromatograms of the final product obtained from EWOD actuation and at macro scale is shown in Fig. 10b. Using the similar approach, Dubois et al also studied the Grieco three-component condensation reaction and found that the reactions in the form of droplets in the EWOD chip are much slower compared to the batch reactions. However, enhancement of the droplet temperature resulted in increasing the reaction kinetics which was almost
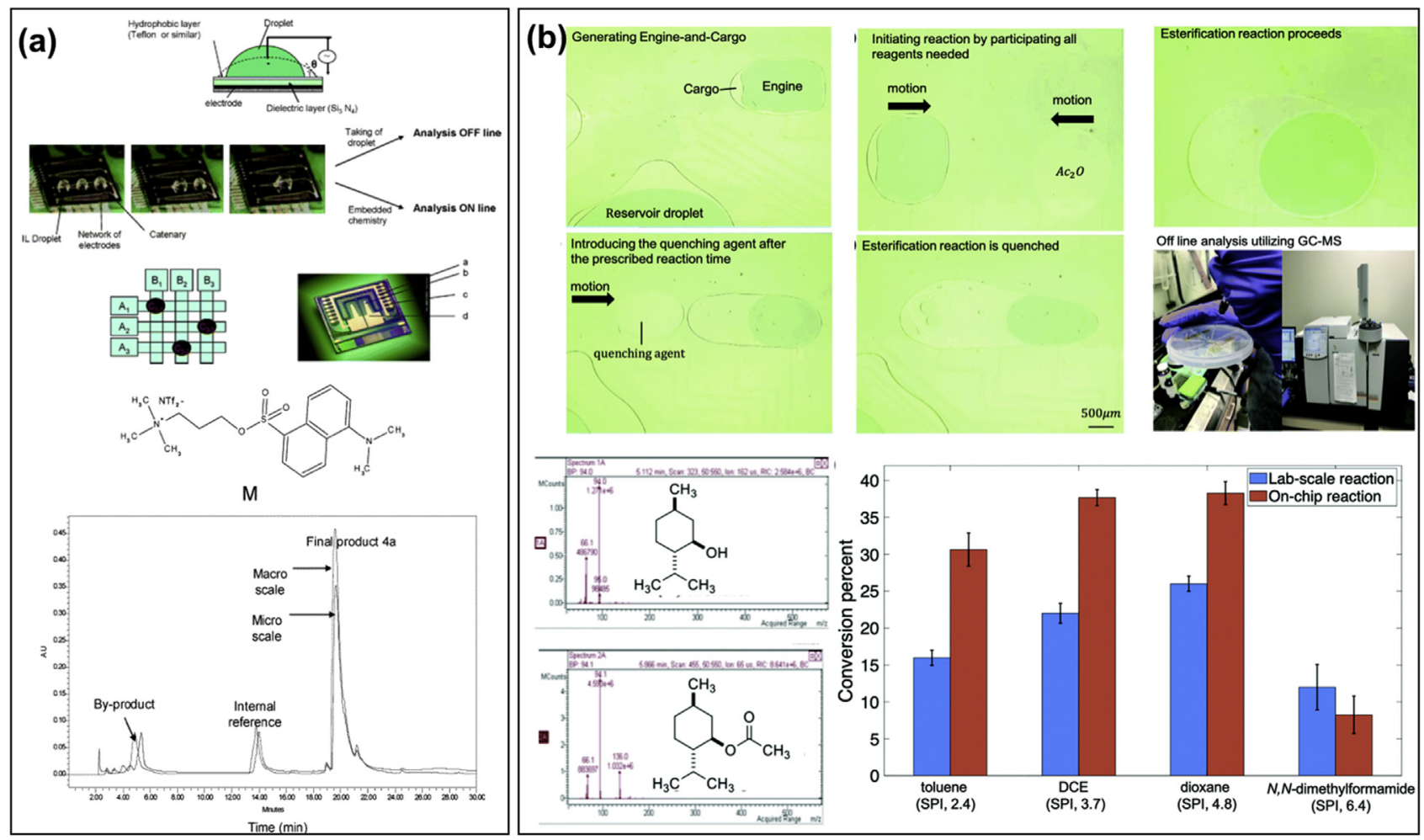

Fig. 10. On-chip chemical-reactions performed on an EWOD chip. (a) Grieco's tetrahydroquinolines synthesis was carried out on the EWOD based DMF chip. The chip design and mechanism of the chemical synthesis based on the EWOD voltage. The comparison of the MALDI-MS results of the reactions performed at macro and micro-scale is also recorded. Reprinted with permission [139]. Copyright 2006, American Chemical Society. (b) Esterification reaction demonstrated on the EWOD device based on the generation of an engine-cargo compound droplet. Steps of the esterification reaction performed on the EWOD device was shown. The obtained MS results confirm the formation of acetylated methanol from methanol suggesting the success of the on-chip esterification reaction. The on-chip esterification reaction of methanol was also performed in the presence of different solvents - toluene, DCE, dioxane and $\mathrm{N}, \mathrm{N}$-dimethylformamide. 
equal to its batch counterparts [143]. Although many EWOD systems have been developed for carrying out chemical reactions, the use of EWOD for organic solvents based reactions is limited. Most of the organic solvents required for chemical reactions possess lower surface tension than water, which hinders their movement in EWOD chips, hence reducing their applicability. To address this unresolved challenge, Torabinia and co-authors developed a new model known as the "engine and cargo" system to electrically actuate the originally nonresponsive organic liquid in the EWOD chip [144]. The design was based on the formation of composite droplets of two immiscible liquids, where the "engine" was defined as a liquid (ionic liquid here) with electrowetting properties and the "cargo" was a non-movable organic solvent (toluene here). Upon application of the actuation voltage, the electrowetting nature of the ionic liquid caused it to dispense from the reservoir channel of the chip; in contrast, toluene remained unaffected. As soon as the ionic liquid droplet approached the toluene droplet, encapsulation of the engine droplet by the cargo occurred, forming a composite droplet. To demonstrate the functionality of EWOD chips for chemical reactions, esterification reactions were chosen as examples due to their significance in organic synthesis, particularly for the synthesis of food preservatives. For the model reaction, the reagent droplet was generated from the reservoir channel and merged with the already generated engine-cargo droplet, followed by mixing and initiation of the esterification reaction (Fig. 10b). The successful formation of the acetylated methanol from methanol after the on-chip esterification reaction was confirmed from the MS analysis (Fig. 10b). The effect of solvents with different polarity index on the esterification reaction was also studied. However, the results show that the polarity of the solvents cannot be correlated to the advancement of the esterification reaction. Furthermore, other reaction parameters such as the reaction kinetics and catalytic loading efficacies were studied in detail in order to prove the potential of EWOD chips as rapid chemical reaction tools. The key advantage of this EWOD driven chemical reaction is that the on-chip reaction kinetics is similar to the off-chip chemical reactions, but as the reaction time increases the standard deviation also increases for the on-chip reactions due to evaporation of the solvent. In addition to the mentioned chemical reactions, EWOD based platforms have been extensively studied for the synthesis of positron emission tomography (PET) tracers for imaging applications $[145,146]$.
The application of electrowetting-driven droplet manipulation is also progressing towards understanding fundamental questions, such as by analysing microbial physiology in their microenvironments, which have important implications for food quality assessment. One such example was published by $\mathrm{Wu}$ et al, who studied physiological processes such as germination of Bacillus atrophaeus endospores in an EWOD platform by detecting in situ conductivity changes [147]. It is important to note that the germination process in endospores is responsible for contamination in food processing facilities; therefore, it is highly critical to monitor the process. For this, a Bacillus atrophaeus endospore droplet was mixed with an Lalanine droplet through EWOD actuation to trigger germination monitored using conductivity quantification. The EWOD chip consisted of an electrode array arranged in a T-junction that was incorporated with two parallel platinum electrodes for conductivity measurements. The results obtained indicated faster germination rates for onchip conductometry measurements than for offchip conductometry due to the larger intensity of the electric field generated from the microelectrode array in the EWOD chip. Unlike the assaybased EWOD systems, this study presented a novel potential of the DMF technique not only for the food industry but also for disease diagnostics.

Apart from aforementioned examples, various groups have studied the on-chip rheological properties using the EWOD device under stress conditions [148]. The rheological properties namely the fluidity, consistency and mechanical characteristics are critical for food processing and quality control since it helps to understand the food texture and the determine the storage time. Nelson et al have fabricated an extensional filament rheometer that can be activated by EWOD device (Fig. 11a). The asfabricated EWOD chip can split the non-wetting samples and induce spreading, thereby creating shear-free liquids that is measured by LED based optical micrometry. EWOD driven break-up test was performed on the samples glycerol and Xanthan gum at an applied voltage of 40-100 VDC for few seconds (Fig. 11b). To carry out the capillary break-up test, the samples was loaded by pipette, followed by generation of a bridge between the sample arms (Fig. 11c). On application of the external voltage, the nonwetting fluids spreads into the chip due to the draining of the liquid bridge. When the shrinkage of the bridge reaches a threshold value to generate an unstable filament, capillary break up takes place. 
(a)
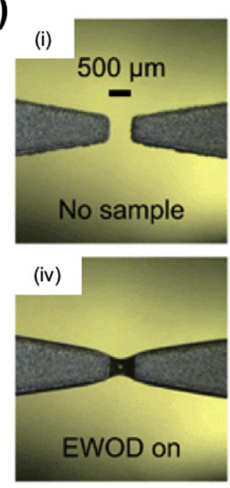

(c)

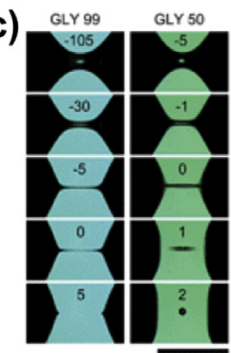

(a)
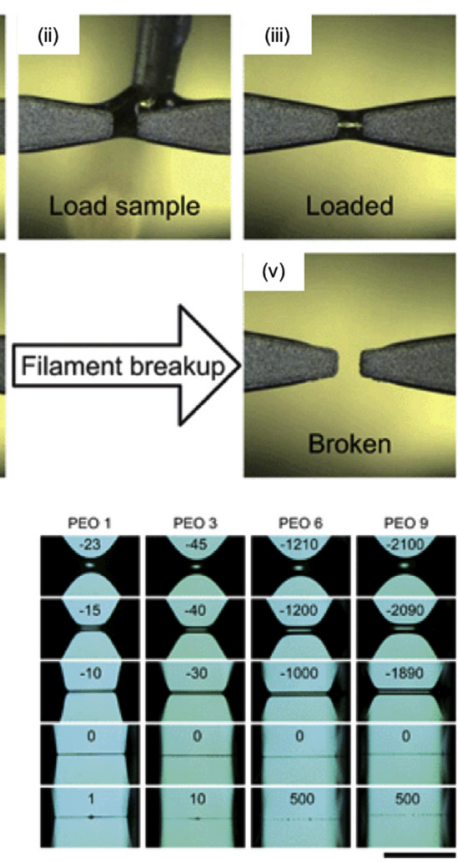

(b) (b)
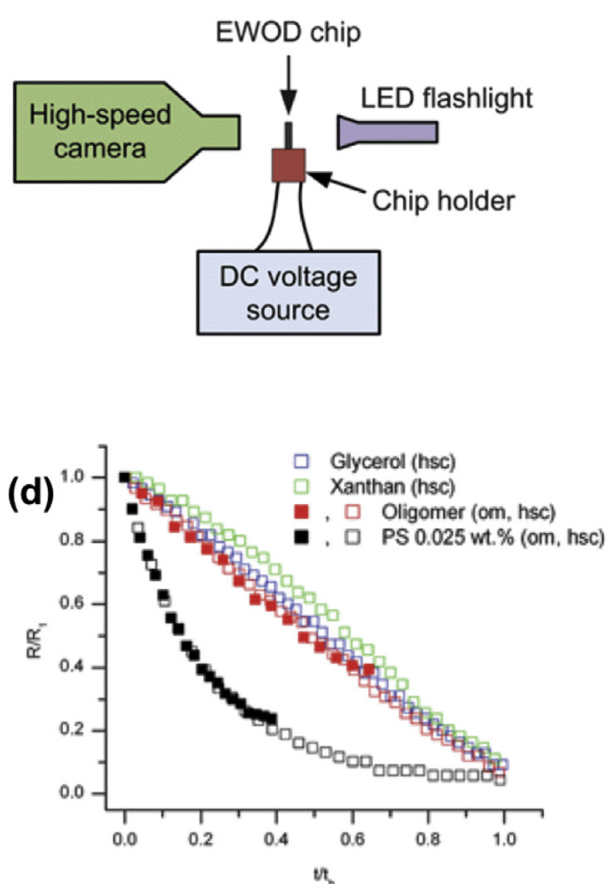

Fig. 11. Rheological analysis of non-wetting liquid samples under break-up conditions in an micro-sized EWOD chip. (a) Break-up analysis under the EWOD actuation showing EWOD driven bridge necking followed by filament break-up conditions. (b) Measurement set-up includes a high-speed camera for break-up step recording. (c) Video images of break-up of glycerol solutions at different frames. (d) Break-up data of various fluids measured by the optical method represented by filled symbols and high-speed camera indicated by hollow symbols. Modified and reprinted with permission [148]. Copyright 2011, Royal Society of Chemistry.

\section{Conclusions and future outlook}

To date, EWOD technology has been a stepping stone in the lab-on-chip paradigm towards performing a broad range of biochemical analytical assays at the micro- and even nanoscale. Several studies have successfully developed multifunctional EWOD devices for on-chip biomedical applications, which have further paved the way for utilization of EWOD for related fields, most importantly food safety and monitoring. Numerous research groups have reported that the electrode geometry and the selection of dielectric material are critical for improving the performance of EWOD devices for detecting food-specific analytes, which are usually present in trace amounts $[54,57,69,79,81]$. Utilizing these advanced design strategies, highly miniaturized and integrated EWOD devices are fabricated, which provides a novel perspective for on-field detection of food samples. In this review, an overview of the recent developments in the field of EWOD-based droplet manipulation processes for the analysis of food contaminants has been provided. The applications of EWOD-based microfluidic devices as portable sample-preparation modules and as sample detection platforms are summarized in this paper. Being fully automated, EWOD systems require very small volumes and no external pumps or actuators and display state-ofthe-art level sensitivity, which makes them promising candidates for commercialization that are capable of replacing mainstream laboratory instruments for detecting chemical and biological analytes in real samples. Despite its remarkable progress, the potential of EWOD for executing entire laboratory assays on chips remains under question. The challenge arises when the target analyte for detection is a biomolecule or an organic compound that consists of complex matrices. Especially for the food sector, the determination of food quality is time-consuming and laborious. In such cases, it is desired to utilize completely automated and programmed miniaturized devices that can facilitate on-chip sample pre-treatment in addition to sample detection in the same platform. To date, very few sample preparation modules based on the EWOD mechanism have been developed, as reported in this paper. Integrating sample preparation and detection in a single EWOD chip is a key aspect for the successful transformation of laboratory-scale 
procedures into a single chip. Significant advances have been made in improving the design of EWOD chips for performing multiplex assays simultaneously; however, the durability of the chips remains a major concern due to the adsorption of the analytes present in food samples on the chip surface. The anti-fouling coating materials currently employed such as SLIPS and pluronics are not robust enough for long-term usage. Therefore, it is highly important to develop novel materials that can completely eliminate the problem of biofouling and make the device reusable. Another challenge associated with EWOD is the high external bias required to actuate the droplets, which is a major roadblock in making the device portable. In most EWOD devices, an AC or DC source is indispensable for droplet manipulation, which is usually bulky and requires high power, hence increasing the cost and limiting the application scope of EWOD. To address these constraints, some research groups have tried to utilize a self powered triboelectric nanogenerator (TENG) as a novel method to power EWOD chips [149-151]. For example, a TENG was developed based on electrospun MXene as the triboelectric layer to power an EWOD chip [151]. In addition, a solid-liquid TENG where water is used as one of the triboelectrification layers has been reported as an efficient power source for EWOD where the droplet energy harvested by the TENG during droplet sliding is used for powering droplet actuation [149]. Because the integration of TENG and EWOD is still in the initial phase of research, there is still much room to explore the parameters governing this integration strategy, especially for solid-liquid TENGs, which can enhance the potential of EWOD for portable applications.

\section{Author contributions}

Conception and design of study: Snigdha Roy Barman, Zong-Hong Lin. Acquisition of data: Snigdha Roy Barman, Subhodeep Chatterjee, Subhajit Saha, Imran Khan. Analysis and interpretation of data: Imran Khan, Snigdha Roy Barman, ZongHong Lin, Dukhyun Choi. Drafting the manuscript: Snigdha Roy Barman, Subhodeep Chatterjee, Imran Khan, Subhajit Saha. Revising the manuscript for important intellectual content: Snigdha Roy Barman, Subhajit Saha, Zong-Hong Lin, Dukhyun Choi, Sangmin Lee. All authors approved the version of manuscript to be published.

\section{Conflict of interest}

The authors declare that no conflicts of interest exist.

\section{Acknowledgements}

The authors would like to thank the Ministry of Science and Technology (MOST 109-2636-E007-013) and the Ministry of Education (MOE 107QR001I5) of Taiwan for financially supporting this research. This research was also supported by the Chung-Ang University Research Grant in 2020.

\section{References}

[1] Malic L, Brassard D, Veres T, Tabrizian M. Integration and detection of biochemical assays in digital microfluidic LOC devices. Lab Chip 2010;10:418-31. https://doi.org/10.1039/ B917668C.

[2] Young EWK, Beebe DJ. Fundamentals of microfluidic cell culture in controlled microenvironments. Chem Soc Rev 2010;39:1036. https://doi.org/10.1039/b909900j.

[3] Ho CMB, Ng SH, Li KHH, Yoon Y-J. 3D printed microfluidics for biological applications. Lab Chip 2015;15: 3627-37. https://doi.org/10.1039/C5LC00685F.

[4] Samiei E, Tabrizian M, Hoorfar M. A review of digital microfluidics as portable platforms for lab-on a-chip applications. Lab Chip 2016;16:2376-96. https://doi.org/ 10.1039/C6LC00387G.

[5] Shakeri A, Jarad NA, Leung A, Soleymani L, Didar TF. Biofunctionalization of Glass- and Paper-Based Microfluidic Devices: A Review. Adv Mater Interfaces 2019;6: 1900940. https://doi.org/10.1002/admi.201900940.

[6] Yeo LY, Chang H-C, Chan PPY, Friend JR. Microfluidic Devices for Bioapplications. Small 2011;7:12-48. https:// doi.org/10.1002/smll.201000946.

[7] Webster A, Greenman J, Haswell SJ. Development of microfluidic devices for biomedical and clinical application. J Chem Technol Biotechnol 2011;86:10-7. https://doi.org/ 10.1002/jctb.2482.

[8] Yang K, Peretz-Soroka H, Liu Y, Lin F. Novel developments in mobile sensing based on the integration of microfluidic devices and smartphones. Lab Chip 2016;16:943-58. https:// doi.org/10.1039/C5LC01524C.

[9] Shen H-H, Fan S-K, Kim C-J, Yao D-J. EWOD microfluidic systems for biomedical applications. Microfluid Nanofluidics 2014;16:965-87. https://doi.org/10.1007/s104040141386-y.

[10] Fair RB. Digital microfluidics: is a true lab-on-a-chip possible? Microfluid Nanofluidics 2007;3:245-81. https:// doi.org/10.1007/s10404-007-0161-8.

[11] Teh S-Y, Lin R, Hung L-H, Lee AP. Droplet microfluidics. Lab Chip 2008;8:198. https://doi.org/10.1039/b715524g.

[12] Zhu P, Wang L. Passive and active droplet generation with microfluidics: a review. Lab Chip 2017;17:34-75. https:// doi.org/10.1039/C6LC01018K.

[13] Mou L, Jiang X. Materials for microfluidic immunoassays: A review. Adv Healthc Mater 2017;6:1601403. https://doi.org/ 10.1002/adhm.201601403.

[14] Zou F, Ruan Q, Lin X, Zhang M, Song Y, Zhou L, et al. Rapid, real-time chemiluminescent detection of DNA mutation based on digital microfluidics and pyrosequencing. 
Biosens Bioelectron 2019;126:551-7. https://doi.org/10.1016/ j.bios.2018.09.092.

[15] de Campos RPS, Rackus DG, Shih R, Zhao C, Liu X, Wheeler AR. "Plug-n-Play" sensing with digital microfluidics. Anal Chem 2019;91:2506-15. https://doi.org/ 10.1021/acs.analchem.8b05375.

[16] Wu B, Ecken S, Swyer I, Li C, Jenne A, Vincent F, et al. Rapid chemical reaction monitoring by digital microfluidics-NMR: Proof of principle towards an automated synthetic discovery platform. Angew Chemie Int Ed 2019; 58:15372-6. https://doi.org/10.1002/anie.201910052.

[17] Myung JH, Hong S. Microfluidic devices to enrich and isolate circulating tumor cells. Lab Chip 2015;15:4500-11. https://doi.org/10.1039/C5LC00947B.

[18] Coelho B, Veigas B, Fortunato E, Martins R, Á guas H, Igreja R, et al. Digital microfluidics for nucleic acid amplification. Sensors 2017;17:1495. https://doi.org/10.3390/ s17071495.

[19] Xi H-D, Zheng H, Guo W, Gañán-Calvo AM, Ai Y, Tsao C$\mathrm{W}$, et al. Active droplet sorting in microfluidics: a review. Lab Chip 2017;17:751-71. https://doi.org/10.1039/ C6LC01435F.

[20] Zhang Y, Nguyen N-T. Magnetic digital microfluidics - a review. Lab Chip 2017;17:994-1008. https://doi.org/10.1039/ C7LC00025A.

[21] Vergauwe N, Witters D, Atalay YT, Verbruggen B, Vermeir S, Ceyssens F, et al. Controlling droplet size variability of a digital lab-on-a-chip for improved bio-assay performance. Microfluid Nanofluidics 2011;11:25-34. https://doi.org/10.1007/s10404011-0769-6.

[22] Pang L, Ding J, Liu X-X, Fan S-K. Digital microfluidics for cell manipulation. TrAC Trends Anal Chem 2019;117:291-9. https://doi.org/10.1016/j.trac.2019.06.008.

[23] Samad MF, Kouzani AZ, Rahman MM, Magniez K, Kaynak A. Design and Fabrication of an Electrode for Lowactuation-Voltage Electrowetting-on-Dielectric Devices. Procedia Technol 2015;20:20-5. https://doi.org/10.1016/ j.protcy.2015.07.005.

[24] Nelson WC, Kim C-J`CJ'. Droplet Actuation by Electrowetting-on-Dielectric (EWOD): A Review. J Adhes Sci Technol 2012:1-25. https://doi.org/10.1163/ $156856111 X 599562$

[25] Lin T-H, Yao D-J. Applications of EWOD Systems for DNA Reaction and Analysis. J Adhes Sci Technol 2012:1-16. https://doi.org/10.1163/156856111X600578.

[26] Byun SH, Yuan J, Yoon MG, Cho SK. Wirelessly powered electrowetting-on-dielectric (EWOD) by planar receiver coils. J Micromechanics Microengineering 2015;25:035019. https://doi.org/10.1088/0960-1317/25/3/035019.

[27] Chen Y, Wang S. Development of Coplanar Electro-Wetting Based Microfluidic Sorter to Select Micro-Particles in High Volume Throughput at Milliliter Amount within Twenty Minutes. Sensors 2018;18:2941. https://doi.org/ $10.3390 / \mathrm{s} 18092941$.

[28] Kim C-J, Loo J, Garrell R, Wheeler A, Moon H. Electrowetting-based microfluidics for analysis of peptides and proteins by matrix-assisted laser desorption/lonization mass spectrometry. Anal Chem 2004;76:4833-8.

[29] Caputo D, de Cesare G, Lo Vecchio N, Nascetti A, Parisi E, Scipinotti R. Polydimethylsiloxane material as hydrophobic and insulating layer in electrowetting-on-dielectric systems. Microelectronics J 2014;45:1684-90. https://doi.org/10.1016/ j.mejo.2014.05.016.

[30] Lee J, Moon H, Fowler J, Schoellhammer T, Kim CJ. Electrowetting and electrowetting on-dielectric for microscale liquid handling. Sensors Actuators, A Phys 2002;95:259-68. https://doi.org/10.1016/S0924-4247(01)00734-8.

[31] Berthier J, Clementz P, Raccurt O, Jary D, Claustre P, Peponnet $\mathrm{C}$, et al. Computer aided design of an EWOD microdevice. Sensors Actuators, A Phys 2006;127:283-94. https://doi.org/10.1016/j.sna.2005.09.026.

[32] Karuwan C, Sukthang K, Wisitsoraat A, Phokharatkul D, Patthanasettakul V, Wechsatol W, et al. Electrochemical detection on electrowetting-on-dielectric digital microfluidic chip. Talanta 2011;84:1384-9. https://doi.org/10.1016 j.talanta.2011.03.073.

[33] Kirby AE, Wheeler AR. Digital Microfluidics: An merging sample preparation platform for Mass Spectroscopy. Anal.Chem. 2013;85:6178-84. https://doi.org/10.1021/ ac401150q.

[34] O'Kennedy R, Leonard P, Hearty S, Daly S, Dillon P, Brennan J, et al. Advances in biosensors for detection of pathogens in food and water. Rapid Methods Biol Chem Contam Food Feed 2005;32:85-104. https://doi.org/10.3920/ 978-90-8686-538-3.

[35] Yang S, Rothman RE. PCR-based diagnostics for infectious diseases: uses, limitations, and future applications in acutecare settings. Lancet Infect Dis 2004;4:337-48. https:// doi.org/10.1016/S1473-3099(04)01044-8.

[36] Rasooly A, Herold KE. Food microbial pathogen detection and analysis using DNA microarray technologies. Foodborne Pathog Dis 2008;5:531-50. https://doi.org/10.1089/ fpd.2008.0119.

[37] de Mello AJ, Beard N. Dealing with real samples: sample pre-treatment in microfluidic systems. Lab Chip 2003;3. https://doi.org/10.1039/b301019h.

[38] Alexandrino M, Grohmann E, Szewzyk U. Optimization of PCR-based methods for rapid detection of Campylobacter jejuni, Campylobacter coli and Yersinia enterocolitica serovar 0:3 in wastewater samples. Water Res 2004;38: 1340-6. https://doi.org/10.1016/j.watres.2003.10.036.

[39] Lazcka O, Campo FJ Del, Muñoz FX. Pathogen detection: A perspective of traditional methods and biosensors. Biosens Bioelectron 2007;22:1205-17. https://doi.org/10.1016/ j.bios.2006.06.036.

[40] Wang D, Wang Z, Chen J, Kinchla AJ, Nugen SR. Rapid detection of Salmonella using a redox cycling-based electrochemical method. Food Control 2016;62:81-8. https:// doi.org/10.1016/j.foodcont.2015.10.021.

[41] Kim JH, Lee JH, Kim JY, Mirzaei A, Wu P, Kim HW, et al Electrowetting on dielectric (EWOD) properties of Tefloncoated electrosprayed silica layers in air and oil media and the influence of electric leakage. J Mater Chem C 2018;6: 6808-15. https://doi.org/10.1039/c8tc01284a.

[42] Cha G, Kim C-J, Ju YS. Thermal conductance switching based on the actuation of liquid droplets through the electrowetting on dielectric (EWOD) phenomenon. Appl Therm Eng 2016;98:189-95. https://doi.org/10.1016/ j.applthermaleng.2015.11.098.

[43] Högnadóttir S, Kristinsson K, Thormar HG, Leosson K Increased droplet coalescence using electrowetting on dielectric (EWOD). Appl Phys Lett 2020;116. https://doi.org/ 10.1063/1.5140202.

[44] Mibus M, Zangari G. Performance and Reliability of Electrowetting-on-Dielectric (EWOD) Systems Based on Tantalum Oxide. ACS Appl Mater Interfaces 2017;9: 42278-86. https://doi.org/10.1021/acsami.7b07366.

[45] Jain V, Raj TP, Deshmukh R, Patrikar R. Design, fabrication and characterization of low cost printed circuit board based EWOD device for digital microfluidics applications. Microsyst Technol 2017;23:389-97. https://doi.org/10.1007/ s00542-015-2680-7.

[46] Mugele F. Fundamental challenges in electrowetting: From equilibrium shapes to contact angle saturation and drop dynamics. Soft Matter 2009;5:3377-84. https://doi.org/ 10.1039/b904493k.

[47] Chen L, Bonaccurso E. Electrowetting - From statics to dynamics. Adv Colloid Interface Sci 2014;210:2-12. https:// doi.org/10.1016/j.cis.2013.09.007.

[48] Li X, Tian H, Shao J, Ding Y, Chen X, Wang L, et al Decreasing the saturated contact angle in electrowettingon-dielectrics by controlling the charge trapping at liquidinterfaces, 2016 Solid interfaces. Adv Funct Mater 2016;26: 2994-3002. https://doi.org/10.1002/adfm.201504705.

[49] Teng P, Tian D, Fu H, Wang S. Recent progress of electrowetting for droplet manipulation: from wetting to 
superwetting systems. Mater Chem Front 2020;4:140-54. https://doi.org/10.1039/C9QM00458K.

[50] Shapiro B, Moon H, Garrell RL, Kim C-J"CJ". Equilibrium behavior of sessile drops under surface tension, applied external fields, and material variations. J Appl Phys 2003;93: 5794-811. https://doi.org/10.1063/1.1563828.

[51] Papathanasiou AG, Boudouvis AG. Manifestation of the connection between dielectric breakdown strength and contact angle saturation in electrowetting. Appl Phys Lett 2005;86:164102. https://doi.org/10.1063/1.1905809.

[52] Narasimhan V, Park S-Y. An Ion Gel as a Low-Cost, SpinCoatable, High-Capacitance Dielectric for Electrowettingon-Dielectric (EWOD). Langmuir 2015;31:8512-8. https:// doi.org/10.1021/acs.langmuir.5b01745.

[53] Guan Y, Tong AY, Nikapitiya NYJB, Moon H. Numerical modeling of microscale droplet dispensing in parallel-plate electrowetting-on-dielectric (EWOD) devices with various reservoir designs. Microfluid Nanofluidics 2016;20:39. https://doi.org/10.1007/s10404-016-1703-8.

[54] Mulla T, Choi S-B. An electromechanical model of an electro-responsive liquid droplet actuator for microsystems: Modeling and verification. Sensors Actuators A Phys 2019; 285:338-47. https://doi.org/10.1016/j.sna.2018.11.031.

[55] Zhang $Q$, Zhang $X$, Zhang $X$, Jiang L, Yin J, Zhang $P$, et al. A feedback-controlling digital microfluidic fluorimetric sensor device for simple and rapid detection of mercury (II) in costal seawater. Mar Pollut Bull 2019;144:20-7. https:// doi.org/10.1016/j.marpolbul.2019.04.063.

[56] Li J, Kim CJ. Current commercialization status of electrowetting-on-dielectric (EWOD) digital microfluidics. Lab Chip 2020;20:1705-12. https://doi.org/10.1039/D0LC00144A.

[57] Cao J, An Q, Liu Z, Jin M, Yan Z, Lin W, et al. Electrowetting on liquid-infused membrane for flexible and reliable digital droplet manipulation and application. Sensors Actuators B Chem 2019;291:470-7. https://doi.org/10.1016/ j.snb.2019.04.102.

[58] Choi K, Ng AHC, Fobel R, Wheeler AR. Digital Microfluidics. Annu Rev Anal Chem 2012;5:413-40. https:// doi.org/10.1146/annurev-anchem-062011-143028.

[59] Ruan Qingyu, Guo Jingjing, YangWang, Zou Fenxiang, Xiaoye Lin W, Yang and C. Digital Microfluidics for Bioanalysis. In: Jiang Xingyu, editor. Nanotechnol. Microfluid. First. Wiley-VCH Verlag GmbH \& Co. KGaA; 2020. p. $47-81$.

[60] Latip ENA, Coudron L, McDonnell MB, Johnston ID, McCluskey DK, Day R, et al. Protein droplet actuation on superhydrophobic surfaces: a new approach toward antibiofouling electrowetting systems. RSC Adv 2017;7: 49633-48. https://doi.org/10.1039/C7RA10920B.

[61] Ruvalcaba-Cardenas AD, Thurgood P, Chen S, Khoshmanesh K, Tovar-Lopez FJ. Droplet on Soft Shuttle: Electrowetting-on-Dielectric Actuation of Small Droplets. ACS Appl Mater Interfaces 2019;11:39283-91. https:// doi.org/10.1021/acsami.9b10796.

[62] Jain V, Hole A, Deshmukh R, Patrikar R. Dynamic capacitive sensing of droplet parameters in a low-cost open EWOD system. Sensors Actuators A Phys 2017;263:224-33. https://doi.org/10.1016/j.sna.2017.06.014.

[63] Ribet F, De Luca E, Ottonello-Briano F, Swillo M, Roxhed N, Stemme G. Zero insertion-loss optical shutter based on electrowetting-on-dielectric actuation of opaque ionic liquid microdroplets. Appl Phys Lett 2019;115:073502. https://doi.org/10.1063/1.5108936.

[64] Yi U-C, Kim C-J"CJ". Soft printing of droplets pre-metered by electrowetting. Sensors Actuators A Phys 2004;114: 347-54. https://doi.org/10.1016/j.sna.2003.12.003.

[65] Hui Y, Peng Z, Alahi MEE, Jiang B, Wu T. Significance of digital microfluidic techniques in biomedical devices for healthcare. In: Assistive Technology for the Elderly. 1st Edition. Academic Press; 2020. p. 281-303. https://doi.org/ 10.1016/B978-0-12-818546-9.00010-5.

[66] Wang J, Chao PH, Hanet S, Van-Dam RM. Performing multi-step chemical reactions in microliter-sized droplets by leveraging a simple passive transport mechanism. Lab Chip 2017;17:4342-55.

[67] Tröls A, Enser H, Jakoby B. Low-cost silver screen-printed electrowetting on dielectrics structure for optofluidic switches. Procedia Eng 2016;168:1061-5. https://doi.org/ 10.1016/j.proeng.2016.11.340.

[68] Hu X, Mibus M, Knospe CR, Zangari G, Reed ML. Impedance spectroscopy and electrical modeling of electrowetting on dielectric devices. J Micromechanics Microengineering 2015;25:045020. https://doi.org/10.1088/09601317/25/4/045020.

[69] Min X, Bao C, Kim WS. Additively Manufactured Digital Microfluidic Platforms for Ion-Selective Sensing. ACS Sensors 2019;4:918-23. https://doi.org/10.1021/ acssensors.8b01689.

[70] Ugsornrat K, Pasakon P, Karuwan C, Sriprachuabwong C, Maturos T, Pogfay T, et al. Low-cost and disposable electrowetting-on-dielectric lab on a chip with an integrated electrochemical detector fabricated by screen-printing process. IEEE Sens J 2019;19:8597-604. https://doi.org/ 10.1109/JSEN.2019.2921789.

[71] Jang I, Ko H, You G, Lee H, Paek S, Chae H, et al. Application of paper EWOD (electrowetting- on-dielectrics) chip: Protein tryptic digestion and its detection using MALDITOF mass spectrometry. BioChip J 2017;11:146-52. https:// doi.org/10.1007/s13206-016-1208-2.

[72] Ma H, Hu S, Jie Y, Jin K, Su Y. A floating top-electrode electrowetting-on- dielectric system. RSC Adv 2020;10: 4899-906. https://doi.org/10.1039/C9RA09491A.

[73] Vergauwe N, Witters D, Ceyssens F, Vermeir S, Verbruggen B, Puers R, et al. A versatile electrowettingbased digital microfluidic platform for quantitative homogeneous and heterogeneous bio-assays. J Micromechanics Microengineering 2011;21:054026. https://doi.org/10.1088/ 0960-1317/21/5/054026.

[74] Jang LS, Hsu CY, Chen CH. Effect of electrode geometry on performance of EWOD device driven by battery-based system. Biomedical Microdevices 2009;11:1029. https:// doi.org/10.1007/s10544-009-9320-x.

[75] Nikapitiya NYJB, Nahar MM, Moon H. Accurate, consistent, and fast droplet splitting and dispensing in electrowetting on dielectric digital microfluidics. Micro and Nano Systems Letters 2017;5:24. https://doi.org/10.1186/s40486017-0058-6.

[76] Samad MF, Kouzani AZ, Hossain MF, Mohammed MI Alam MNH. Reducing electrowetting-on-dielectric actuation voltage using a novel electrode shape and a multilayer dielectric coating. Microsystem Technologies 2017;23: 3005-13. https://doi.org/10.1007/s00542-016-3087-9samad 2017.

[77] Chen J, Yu Y, Li J, Lai Y, Zhou J. Size-variable droplet actuation by interdigitated electrowetting electrode101; 2012. p. 234102. https://doi.org/10.1063/1.4769433.

[78] Kalsi S, Valiadi M, Tsaloglou M-N, Parry-Jones L, Jacobs A, Watson $\mathrm{R}$, et al. Rapid and sensitive detection of antibiotic resistance on a programmable digital microfluidic platform. Lab Chip 2015;15:3065-75. https://doi.org/10.1039/ C5LC00462D.

[79] Schertzer MJ, Ben-Mrad R, Sullivan PE. Using capacitance measurements in EWOD devices to identify fluid composition and control droplet mixing. Sensors Actuators B Chem 2010;145:340-7. https://doi.org/10.1016/ j.snb.2009.12.019.

[80] Gao A, Liu X, Li T, Zhou P, Wang Y, Yang Q, et al. Digital microfluidic chip for rapid portable detection of mercury(II). IEEE Sens J 2011;11:2820-4. https://doi.org/10.1109/ JSEN.2011.2170969.

[81] Petralia S, Motta D, Conoci S. EWOD silicon biosensor for multiple nucleic acids analysis. Biotechnol Bioeng 2019;116: 2087-94. https://doi.org/10.1002/bit.26987.

[82] Coudron L, McDonnell MB, Munro I, McCluskey DK, Johnston ID, Tan CKL, et al. Fully integrated digital microfluidics platform for automated immunoassay; A 
versatile tool for rapid, specific detection of a wide range of pathogens. Biosens Bioelectron 2019;128:52-60. https:// doi.org/10.1016/j.bios.2018.12.014.

[83] Moon H, Wheeler AR, Garrell RL, Loo JA, Kim C-J “CJ”. An integrated digital microfluidic chip for multiplexed proteomic sample preparation and analysis by MALDI-MS. Lab Chip 2006;6:1213. https://doi.org/10.1039/b601954d.

[84] Prakash R, Pabbaraju K, Wong S, Wong A, Tellier R, Kaler K. Multiplex, quantitative, reverse transcription PCR detection of influenza viruses using droplet microfluidic technology. Micromachines 2014;6:63-79. https://doi.org/ 10.3390/mi6010063.

[85] Clement CE, Thio SK, Park S-Y. An optofluidic tunable Fresnel lens for spatial focal control based on electrowetting-on-dielectric (EWOD). Sensors Actuators B Chem 2017;240:909-15. https://doi.org/10.1016/j.snb.2016.08.125.

[86] Brassard D, Malic L, Miville-Godin C, Normandin F, Veres T. Advanced EWOD-based digital microfluidic system for multiplexed analysis of biomolecular interactions. In: 2011 IEEE 24th Int. Conf. Micro Electro Mech. Syst., IEEE; 2011. p. 153-6. https://doi.org/10.1109/ MEMSYS.2011.5734384.

[87] Cheng W, Liu J, Zheng Z, He X, Zheng B, Zhang H, et al. Adaptive optical beam steering and tuning system based on electrowetting driven fluidic rotor. Commun Phys 2020;3:25. https://doi.org/10.1038/s42005-020-0294-6.

[88] Geng H, Cho SK. Antifouling digital microfluidics using lubricant infused porous film. Lab Chip 2019;19:2275-83. https://doi.org/10.1039/C9LC00289H.

[89] Jebrail MJ, Bartsch MS, Patel KD. Digital microfluidics: a versatile tool for applications in chemistry, biology and medicine. Lab Chip 2012;12:2452. https://doi.org/10.1039/ c21c40318h.

[90] Hird SJ, Lau BPY, Schuhmacher R, Krska R. Liquid chromatography-mass spectrometry for the determination of chemical contaminants in food. TrAC Trends Anal Chem 2014;59:59-72. https://doi.org/10.1016/j.trac.2014.04.005.

[91] Zhao X, Lin C-W, Wang J, Oh DH. Advances in rapid detection methods for foodborne pathogens. J Microbiol Biotechnol 2014;24:297-312. https://doi.org/10.4014/ jmb.1310.10013.

[92] Baeumner AJ. Biosensors for environmental pollutants and food contaminants. Anal Bioanal Chem 2003;377:434-45. https://doi.org/10.1007/s00216-003-2158-9.

[93] Hanna SE, Connor CJ, Wang HH. Real-time polymerase chain reaction for the food microbiologist: technologies, Applications, and limitations. J Food Sci 2006;70:R49-53. https://doi.org/10.1111/j.1365-2621.2005.tb07149.x.

[94] Zhang T, Fang HHP. Applications of real-time polymerase chain reaction for quantification of microorganisms in environmental samples. Appl Microbiol Biotechnol 2006;70: 281-9. https://doi.org/10.1007/s00253-006-0333-6.

[95] Salihah NT, Hossain MM, Lubis H, Ahmed MU. Trends and advances in food analysis by real-time polymerase chain reaction. J Food Sci Technol 2016;53:2196-209. https:// doi.org/10.1007/s13197-016-2205-0.

[96] Regan JF, Makarewicz AJ, Hindson BJ, Metz TR, Gutierrez DM, Corzett TH, et al. Environmental monitoring for biological threat agents using the autonomous pathogen detection system with multiplexed polymerase chain reaction. Anal Chem 2008;80:7422-9. https://doi.org/10.1021/ ac801125x.

[97] Foat TG, Sellors WJ, Walker MD, Rachwal PA, Jones JW, Despeyroux DD, et al. A prototype personal aerosol sampler based on electrostatic precipitation and electrowetting-on-dielectric actuation of droplets. J Aerosol Sci 2016;95:43-53. https://doi.org/10.1016/j.jaerosci.2016.01.007.

[98] Zhao Y, Cho SK. Microparticle sampling by electrowettingactuated droplet sweeping. Lab Chip 2006;6:137-44. https:// doi.org/10.1039/b511645g.

[99] Huang PW, Wang TT, Lin SW, Chang YC, Fan SK. Dielectrophoretic Cell Concentrator on EWOD-Based Chips.
In: 2006 1st IEEE International Conference on Nano/Micro Engineered and Molecular Systems; 2006. p. 1418-21. https://doi.org/10.1109/NEMS.2006.334779.

[100] Lejard-Malki R, Follet J, Vlandas A, Senez V. Selective electrohydrodynamic concentration of waterborne parasites on a chip. Lab Chip 2018;18:3310-22. https://doi.org/ 10.1039/C8LC00840J.

[101] Cho SK, Zhao Y, Kim CJC. Concentration and binary separation of micro particles for droplet-based digital microfluidics. Lab Chip 2007;7:490-8. https://doi.org/10.1039/ b615665g.

[102] Lee H, Lee S, Jang I, Kim J, You G, Kim E, et al. Sample preparation of chemical warfare agent simulants on a digital microfluidic (DMF) device using magnetic bead based solid-phase extraction. Microfluid Nanofluidics 2017;21:141. https://doi.org/10.1007/s10404-017-1976-6.

[103] Wheeler AR, Moon H, Kim CJC, Loo JA, Garrell RL. Electrowetting-based microfluidics for analysis of peptides and proteins by matrix-assisted laser desorption/ionization mass spectrometry. Anal Chem 2004;76:4833-8. https:// doi.org/10.1021/ac0498112.

[104] Nelson WC, Peng I, Lee GA, Loo JA, Garrell RL, Kim CJ. Incubated Protein Reduction and Digestion on an Electrowetting-on-Dielectric Digital Microfluidic Chip for MALDIMS. Anal Chem 2010;82:9932-7. https://doi.org/10.1021/ ac101833b.

[105] Gorbatsova J, Borissova M, Kaljurand M. Electrowettingon-dielectric actuation of droplets with capillary electrophoretic zones for off-line mass spectrometric analysis. J Chromatogr A 2012;1234:9-15. https://doi.org/10.1016/ i.chroma.2011.12.052.

[106] Wijethunga PAL, Nanayakkara YS, Kunchala P, Armstrong DW, Moon H. On- chip drop-to-drop liquid microextraction coupled with real-time concentration monitoring technique. Anal. Chem. 2011;83:1658-64. https://doi.org/10.1021/ac102716s.

[107] Paul S, Moon H. In separation of binary solution by liquidliquid microextraction on EWOD digital microfluidics. In: 2017 IEEE 12th International Conference on Nano/Micro Engineered and Molecular Systems (NEMS); 2017. p. 342-5. https://doi.org/10.1109/NEMS.2017.8017038.

[108] Kojima T, Lin C, Takayama S, Fan S. determination of aqueous two-phase system binodals and tie-lines by electrowetting-on-dielectric droplet manipulation. cbic.201800553 ChemBioChem 2018. https://doi.org/ 10.1002/cbic.201800553.

[109] Liu CW, Lin CC, Chen LC, Fan SK, Chang HC. An affinity sensor improved by ewod actuator-based microfluidic chip. Biomedical Engineering: Applications, Basis and Communications 2009;21:461-5. https://doi.org/10.4015/ S1016237209001659liu 2019.

[110] Huang Y, Peng Y, Fan S. Microfluidic immunosensor based on insulator dielectrophoresis and electrowetting-ondielectric. In: 2010 IEEE 5th International Conference on Nano/Micro Engineered and Molecular Systems, Xiamen; 2010. p. 337339. https://doi.org/10.1109/NEMS.2010.5592229.

[111] Mashraei Y, Sivashankar S, Buttner U, Salama KN. Integration of fractal biosensor in a digital microfluidic platform. IEEE Sensors Journal 2016;16:8775-83. https:// doi.org/10.1109/JSEN.2016.2578440.

[112] Klein D. Quantification using real-time PCR technology: applications and limitations. Trends Mol Med 2002;8 257-60. https://doi.org/10.1016/S1471-4914(02)02355-9.

[113] Law JW-F, Ab Mutalib N-S, Chan K-G, Lee L-H. Rapid methods for the detection of foodborne bacterial pathogens: principles, applications, advantages and limitations. Front Microbiol 2015;5:215-25. https://doi.org/10.3389/ fmicb.2014.00770.

[114] Chang Y-H, Lee G-B, Huang F-C, Chen Y-Y, Lin J-L. Integrated polymerase chain reaction chips utilizing digital microfluidics. Biomed Microdevices 2006;8:215-25. https:// doi.org/10.1007/s10544-006-8171-y. 
[115] Sista R, Hua Z, Thwar P, Sudarsan A, Srinivasan V, Eckhardt A, et al. Development of a digital microfluidic platform for point of care testing. Lab Chip 2008;8:2091. https://doi.org/10.1039/b814922d.

[116] Barbulovic-Nad I, Yang H, Park PS, Wheeler AR. Digital microfluidics for cell-based assays. Lab Chip 2008;8:519-26. https://doi.org/10.1039/b717759c.

[117] Hua Z, Rouse JL, Eckhardt AE, Srinivasan V, Pamula VK, Schell WA, et al. Multiplexed Real-Time Polymerase Chain Reaction on a Digital Microfluidic Platform. Anal Chem 2010;82:2310-6. https://doi.org/10.1021/ac902510u.

[118] Delattre C, Allier CP, Fouillet Y, Jary D, Bottausci F, Bouvier D, et al. Macro to microfluidics system for biological environmental monitoring. Biosens Bioelectron 2012;36: 230-5. https://doi.org/10.1016/j.bios.2012.04.024.

[119] Chang YH, Lee GB, Huang FC, Chen YY, Lin JL. Integrated polymerase chain reaction chips utilizing digital microfluidics. Biomedical Microdevices 2006;8:215-25. https:// doi.org/10.1007/s10544-006-8171-y.

[120] Ugsornrat $K$, Maturus $T$, Jomphoak A, Pogfai $T$, Afzulpurkar NV, Wisitsoraat A, et al. In simulation and experimental study of electrowetting on dielectric (EWOD) device for a droplet based polymerase chain reaction system. In: 13th International Conference on Biomedical Engineering; 2009. p. 859-62. https://doi.org/10.1007/978-3540-92841-6_211.

[122] Koo CKW, He F, Nugen SR. An inkjet-printed electrowetting valve for paper-fluidic sensors. Analyst 2013;138: 4998-5004. https://doi.org/10.1039/c3an01114c.

[123] Hadwen B, Broder GR, Morganti D, Jacobs A, Brown C, Hector JR, Kubota Y, Morgan H. Programmable large area digital microfluidic array with integrated droplet sensing for bioassays. Lab Chip 2012;12:3305-13. https://doi.org/ 10.1039/C2LC40273D.

[124] Shin YJ, Lee JB. In digital microfluidics-based highthroughput imaging for systems biology. Sensors, IEEE 2008:1202-5. https://doi.org/10.1109/ICSENS.2008.4716658.

[125] Quang DT, Kim JS. Fluoro- and chromogenic chemodosimeters for heavy metal ion detection in solution and biospecimens. Chem Rev 2010;110:6280-301. https:// doi.org/10.1021/cr100154p.

[126] Farooq U, Kozinski JA, Khan MA, Athar M. Biosorption of heavy metal ions using wheat based biosorbents - A review of the recent literature. Bioresour Technol 2010;101: 5043-53. https://doi.org/10.1016/j.biortech.2010.02.030.

[127] Li M, Gou H, Al-Ogaidi I, Wu N. Nanostructured sensors for detection of heavy metals: A review. ACS Sustain Chem Eng 2013;1:713-23. https://doi.org/10.1021/sc400019a.

[128] Saidur MR, Aziz ARA, Basirun WJ. Recent advances in DNA-based electrochemical biosensors for heavy metal ion detection: A review. Biosens Bioelectron 2017;90:125-39. https://doi.org/10.1016/j.bios.2016.11.039.

[129] Bansod B, Kumar T, Thakur R, Rana S, Singh I. A review on various electrochemical techniques for heavy metal ions detection with different sensing platforms. Biosens Bioelectron 2017;94:443-55. https://doi.org/10.1016/ j.bios.2017.03.031.

[130] Gumpu MB, Sethuraman S, Krishnan UM, Rayappan JBB. A review on detection of heavy metal ions in water - An electrochemical approach. Sensors Actuators B Chem 2015; 213:515-33. https://doi.org/10.1016/j.snb.2015.02.122.

[131] Ye B-C, Yin B-C. Highly sensitive detection of mercury(II) Ions by fluorescence polarization enhanced by gold nanoparticles. Angew Chemie Int Ed 2008;47:8386-9. https:// doi.org/10.1002/anie.200803069.

[132] Pandey SK, Kim K-H, Brown RJC. Measurement techniques for mercury species in ambient air. TrAC Trends Anal Chem 2011;30:899-917. https://doi.org/10.1016/ j.trac.2011.01.017.

[133] Ma W, Sun M, Xu L, Wang L, Kuang H, Xu C. A SERS active gold nanostar dimer for mercury ion detection. Chem Commun 2013;49:4989. https://doi.org/10.1039/c3cc39087j.
[134] Xing C, Liu L, Zhang X, Kuang H, Xu C. Colorimetric detection of mercury based on a strip sensor. Anal Methods 2014;6:6247-53. https://doi.org/10.1039/C3AY42002G.

[135] Atalay YT, Vermeir S, Witters D, Vergauwe N, Verbruggen B, Verboven P, et al. Microfluidic analytical systems for food analysis. Trends Food Sci Technol 2011;22: 386-404. https://doi.org/10.1016/j.tifs.2011.05.001.

[136] Zhang Y, Zuo P, Ye B-C. A low-cost and simple paperbased microfluidic device for simultaneous multiplex determination of different types of chemical contaminants in food. Biosens Bioelectron 2015;68:14-9. https://doi.org/ 10.1016/j.bios.2014.12.042.

[137] Busa L, Mohammadi S, Maeki M, Ishida A, Tani H Tokeshi M. Advances in microfluidic paper-based analytical devices for food and water analysis. Micromachines 2016;7:86. https://doi.org/10.3390/mi7050086.

[138] Meziane F, Raimbault V, Hallil H, Joly S, Conédéra V, Lachaud JL, et al. Study of a polymer optical microring resonator for hexavalent chromium sensing. Sensors Actuators B Chem 2015;209:1049-56. https://doi.org/10.1016/ j.snb.2014.11.101.

[139] Dubois P, Marchand G, Fouillet $Y$, Berthier J, Douki T, Hassine $\mathrm{F}$, et al. Ionic Liquid Droplet as e-Microreactor. Anal Chem 2006;78:4909-17. https://doi.org/10.1021/ ac060481q.

[140] Ding H, Sadeghi S, Shah GJ, Chen S, Keng PY, Kim C$\mathrm{J}$ "CJ,", et al. Accurate dispensing of volatile reagents on demand for chemical reactions in EWOD chips. Lab Chip 2012;12:3331. https://doi.org/10.1039/c2lc40244k.

[141] Javed MR, Chen S, Kim H-K, Wei L, Czernin J, Kim C-JC, et al. Efficient radiosynthesis of 3'-deoxy-3'-18f-fluorothymidine using electrowetting-on dielectric digital microfluidic chip. J Nucl Med 2014;55:321-8. https:// doi.org/10.2967/jnumed.113.121053.

[142] Casalini S, Berto M, Bortolotti CA, Foschi G, Operamolla A, Lauro $\mathrm{M} \mathrm{Di}$, et al. Electrowetting of nitro-functionalized oligoarylene thiols self-assembled on polycrystalline gold, 2015 electrowetting of nitro-functionalized oligoarylene thiols self-assembled on polycrystalline gold. ACS Appl Mater Interfaces 2015;7:3902-9. https://doi.org/10.1021/ am509104z.

[143] Dubois P, Marchand G, Gmouh S, Vaultier M. Reaction rates as a function of scale within ionic liquids: microscale in droplet microreactors versus macroscale reactions in the case of the grieco three-component condensation reaction 2007;13:5642-8. chem.200700331dubois2007.

https://doi.org/10.1002/

[144] Torabinia M, Asgari P, Dakarapu US, Jeon J, Moon H. Onchip organic synthesis enabled using an engine-and-cargo system in an electrowetting-on-dielectric digital microfluidic device. Lab Chip 2019;19:3054-64. https://doi.org/ 10.1039/C9LC00428A.

[145] Wang YB, Su LY, Fu CS, Huang CS, Hsu W. Droplet-based label-free detection system based on guided-mode resonance and electrowetting-on-dielectric for concentration measurement. Jpn J Appl Phy 2017;56:050313.

[146] Kim HK, Chen S, Javed MR, Lei J, Kim CJ, Keng PY, Dam MV. Multi-step organic synthesis of four different Molecular probes in digital microfluidic device. In: Proceedings of the $16^{\text {th }}$ International Conference on Miniaturized Systems for Chemistry and Life Sciences; 2012. p. 617-9.

[147] Wu WJ, Liu SL, Yung PT. Realization of conductometry on a digital microfluidic platform for real-time monitoring of Bacillus atrophaeus endospore germination. IEEE Sens J 2016;16:2244-50. https://doi.org/10.1109/JSEN.2016.2521411.

[148] Nelson WC, Kavehpour HP, Kim CJC. A miniature capillary breakup extensional rheometer by electrostatically assisted generation of liquid filaments. Lab Chip 2011;11:2424-31. https://doi.org/10.1039/C0LC00691BNeson 2011.

[149] Chen G, Liu X, Li S, Donga M, Jiang D. A droplet energy harvesting and actuation system for self-powered digital 
microfluidics. Lab Chip 2018;18:1026-34. https://doi.org/ 10.1039/C7LC01259D.

[150] Kim W, Choi D, Kwon JY, Choi D. A self-powered triboelectric microfluidic system for liquid sensing. J. Mater. Chem. A 2018;6:14069-76. https://doi.org/10.1039/ C8TA04546A.
[151] Jiang C, Wua C, Li X, Yao Y, Lan L, Zhao F, Ye Z, Ying Y, Ping J. All-electrospun flexible triboelectric nanogenerator based on metallic MXene nanosheets. Nano Energy 2019;59: 268-76. https://doi.org/10.1016/j.nanoen.2019.02.052. 